\title{
A Survey and Analysis on Automated Glioma Brain Tumor Segmentation and Overall Patient Survival Prediction
}

This paper was downloaded from TechRxiv (https://www.techrxiv.org).

LICENSE

CC BY-SA 4.0

SUBMISSION DATE / POSTED DATE

26-01-2021 / 27-01-2021

\section{CITATION}

Agravat, Rupal; Raval, Mehul (2021): A Survey and Analysis on Automated Glioma Brain Tumor Segmentation and Overall Patient Survival Prediction. TechRxiv. Preprint. https://doi.org/10.36227/techrxiv.13642904.v1

$\mathrm{DOI}$

10.36227/techrxiv.13642904.v1 


\title{
A Survey and Analysis on Automated Glioma Brain Tumor Segmentation and Overall Patient Survival Prediction
}

\author{
Rupal R. Agravat • Mehul S. Raval
}

Received: date / Accepted: date

\begin{abstract}
Glioma is the most deadly brain tumor with high mortality. Treatment planning by human experts depends on the proper diagnosis of physical symptoms along with Magnetic Resonance(MR) image analysis. Highly variability of a brain tumor in terms of size, shape, location, and a high volume of MR images makes the analysis time-consuming. Automatic segmentation methods achieve a reduction in time with excellent reproducible results. The article aims to survey the advancement of automated methods for Glioma brain tumor segmentation. It is also essential to make an objective evaluation of various models based on the benchmark. Therefore, the 2012 - 2019 BraTS challenges databa evaluates state-of-the-art methods. The complexity of tasks under the challenge has grown from segmentation (Task1) to overall survival prediction (Task 2) to uncertainty prediction for classification (Task 3 ). The paper covers the complete gamut of brain tumor segmentation using handcrafted features to deep neural network models for Task 1 . The aim is to showcase a complete change of trends in automated brain tumor models. The paper also covers end to end joint models involving brain tumor segmentation and overall survival prediction. All the methods are probed, and parameters that affect performance are tabulated and analyzed.
\end{abstract}

Rupal R. Agravat

School of Engineering and Applied Science,

Ahmedabad University,

Ahmedabad, India.

E-mail: rupal.agravat@iet.ahduni.edu.in

Mehul S. Raval

School of Engineering and Applied Science,

Ahmedabad University,

Ahmedabad, India.

E-mail: mehul.raval@ahduni.edu.in
Keywords Deep Learning - Medical Image Analysis . Brain Tumor Segmentation · Magnetic Resonance Imaging · Overall Survival Prediction

\section{Introduction}

From the days the medical images are captured through the imaging devices and digitally preserved, researchers have started to build computerized automated and semiautomated analysis techniques to address a variety of problems like detection, segmentation, classification, and registration. Till 1990s, medical image analysis was done with pixel processing techniques to detect edge/line with filters, regions based on the similarity of pixels or fit mathematical models to detect lines/elliptical shapes. Afterwards, shape models, atlas models and probabilistic models had become successful for medical image analysis, where from the observations(training data) the model learns to predict the unseen data. This trend had moved towards the machine learning models where features are extracted from the data and fed into the computer to make it learn the underlying class/pattern as per the input features and prediction of unseen data is made in the future. This approach has changed the trend of human-dependent system to machine dependent system where the machine learns from the example data. Such algorithms works very well with the high dimensional feature space to find the optimal decision boundary. Here the only thing which is not done by computer is the feature extraction, which leads to the era of deep learning where the computer learn the optimal set of features for the problem at hand. The deep learning models transform the input data from image/audio/video/text to output data which is location/presence/spread and incrementally learns high di- 
mensional features with the help of set of intermediate layers between input and output layers. Medical image analysis has reached the extent where such algorithms play a significant role in the early detection of the disease based on the initial symptoms leading to better treatments. The deadly disease like cancer(Glioma-the cancerous brain tumor), if detected in the early stage, can increase the life expectancy.

\subsection{Brain Tumors and Their Types}

When the natural cycle of the tissues in the brain breaks and growth becomes uncontrollable, it results in a brain tumor[56]. The brain tumor is of two types, (1) primary and (2) secondary. The primary tumor starts with the brain tissues and grows within the brain, whereas secondary tumor spreads in the brain and from the other cancerous organs[24]. More than 100 types of brain tumors are named based on the tissue and the brain part where it starts to grow. Out of all these tumors, Glioma is the most life-threatening brain tumor. It occurs in the glial cells of the brain. The severity grades of the Glioma tumors depends on[115].

- the tumorous cell growth rate.

- blood supply to the tumorous cells.

- presence of necrosis (dead cells at the center of the tumor).

- location of the tumor within the brain.

- confined area of the tumor.

- its structural similarity with the healthy cells.

Grade I and II, are known as Low-Grade Glioma (LGG), which are benign tumors. Grade III and IV are known as High-Grade Glioma (HGG), which are malignant tumors. When symptoms like nausea, vomiting, fatigue, loss of sensation or movement, difficulty in balancing, persist for the longer duration, it is advisable to go through the imaging screening to know the internal structural changes in the brain due to tumor.

\subsection{Brain Imaging Modalities}

Various imaging techniques are used for brain tumor screening which includes positron emission tomography(PET), Computed Tomography(CT) and Magnetic Resonance Imaging(MRI). In PET, the radioactive tracer is injected in the body, which captures the high level of chemical activities of the disease infected body part. In CT, the X-ray tube rotates around the patient's body and emits a narrow X-ray beam, which is passed through the patient body to generate cross-sectional images of the brain. MRI uses a strong magnetic field around the patient's body, which aligns the protons in the body to the generated magnetic field, which is followed by the passing of radiofrequency signals to the body. When the current is off, the protons emit the energy and tries to align with the magnetic field. The emitted energy form an image which records the response of various tissues of the brain. There are two types of MRI: 1)fMRI(Functional MRI): It measures the brain activities from the change in the blood flow, 2) sMRI(Structural MRI): It captures the anatomy and pathology of the brain. The proposed article uses the sMRI as the focus to deal with the pathology in the brain. Various modalities of sMRI captures responses of the tissues which leads to distinct biological information in the images. Various modalities of sMRI are:

- Diffusion Weighted Image(DWI) MRI: MR imaging technique measuring the diffusion of water molecules within tissue voxels. DWI is often used to visualize hyperintensities.

- FLAIR MRI:an MRI pulse sequence which suppresses the fluid (mainly cerebrospinal fluid (CSF)) and enhances edema.

- T1w MRI:basic MRI pulse sequence that captures longitudinal relaxation time (time constant required for excited protons to return to equilibrium) differences of tissues.

- T1Gd MRI:a contrast enhancing agent, Gadolinium is injected into the body and after that $\mathrm{T} 1 \mathrm{se}-$ quence is acquired. This contrast enhancing agent shortens the T1 time which results in bright appearance of blood vessels and pathologies like tumor.

- T2w MRI:basic MRI pulse sequence that captures transverse relation time(T2) differences of tissues.

In general for brain tumors, CT and MRI are the commonly used techniques. Both the imaging techniques are essential, and the differences between CT and MRI are listed in Table 1. Fig. 1 shows the imaging difference between CT and MRI, along with its various modalities. Healthy brain tissues are of three classes: 1) Gray Matter(GM), 2) White Matter (WM), and 3) Cerebrospinal Fluid(CSF). Such soft tissue detailing is captured clearly in MRI compared to CT.

Further different MRI modalities capture responses of various brain tissues differently. The tumor adds one more tissue class in the brain. Fig. 2 shows the difference in the appearance of tumor in CT and MRI.

Table 2 shows the intensity variation of the tumor in different MRI modalities. The tumor class overlaps the normal tissue intensities, e.g., in T1 MR images GM, CSF, and tumor appear to be dark, whereas, in T2 GM, CSF and tumor appear to be bright. It is desirable to use the combination of various modality MRI images for 
Table 1: Difference between CT and MRI[93].

\begin{tabular}{l} 
Advantages and \\
CT \\
\hline Non-invasive. \\
Fast. \\
Cheap. \\
Less accurate. \\
Coarse tissue details. \\
Single modality
\end{tabular}

Generates 3D images.

Images can be in axial, coro- Images can be in axial, coronal and sagittal views.

\section{Risks \\ CT}

Harms unborn babies.

MRI

A small dosage of radiation.

Reacts with the metals in the body due to magnetic field.

The loud noise of the machine causes hearing issues.

Reacts to the use of dyes. Increases body temperature when exposed for a longer duration.

Imaging difficulties in case of claustrophobia.

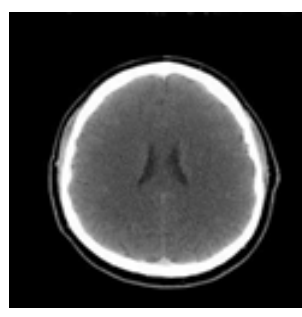

(a)

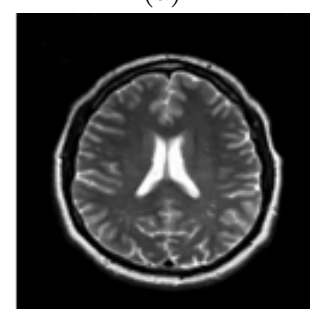

(c)

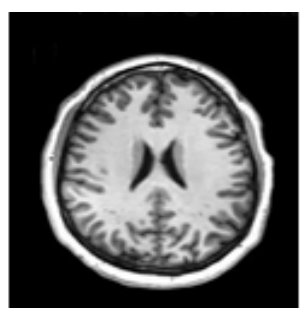

(b)

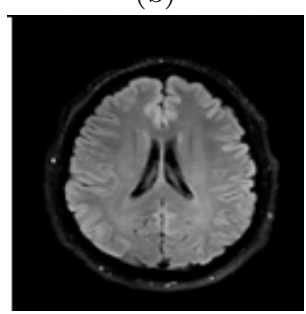

(d)
Fig. 1: Imaging modalities[55] a) CT b) T1 MRI c) T2 MRI and d) DWI MRI.

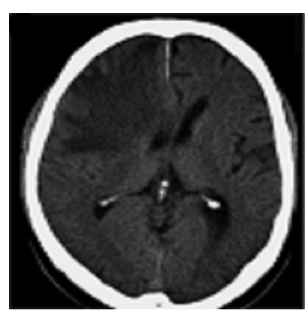

(a)

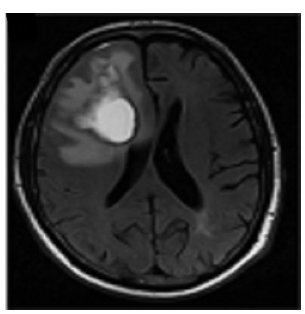

(b)
Fig. 2: Appearance of Tumor in a) CT and b) MRI[66].

the analysis purpose[4]. The rest of the article focuses on the methods based on MR images.

Table 2: The appearance of Normal Brain Tissues and Tumor in various MRI modalities.

\begin{tabular}{lllll}
\hline & GM & WM & CSF & Tumor \\
\hline T1 & Dark & White & Dark & Dark \\
T2 & Light & Dark & White & Bright \\
T1c & Dark & White & Dark & Bright \\
FLAIR & Light & Dark & Dark & Bright \\
\hline
\end{tabular}

The availability of the benchmark dataset has boosted the research in the area of computer-assisted analysis for brain tumor segmentation. Various types of methods for the segmentation task includes semi-automated and automated methods. The semi-automated methods require user input to initiate the process. Circular automata and other random field methods require seed point, diameter, or rough boundary selection for further computation. Atlas-based methods try to fit the pathological image with a healthy image to locate the abnormal brain area. Pathological atlas creation is another approach to determine the abnormality in the brain. Expectation maximization methods iteratively refine the categories of the brain voxels from the input of Gaussian Mixture Models or atlas. The automated methods include machine learning methods, which use the features of the image for voxel classification. Later on, the deep learning methods, specifically Convolution Neural Network(CNN) based methods, have shown success in the field of semantic segmentation, and such methods are adopted widely for brain tumor segmentation. The CNN methods with various architectures as well as ensemble approaches have proven to be the best methods for the segmentation task. In addition to the segmentation task, the survival prediction task predicts the survival days of patients. The contribution of the paper is as follows: 
1. It is the most exhaustive review covering brain tumor imaging modalities, challenges in medical image analysis, evaluation metrics, BraTS dataset evolution, Pre-processing and post processing methods, segmentation methods, proposed architecture, hardware and software for implementation, overall survival predictions, and limitations.

2. It exhaustively traces the development in brain tumor segmentation by covering the models based on handcrafted features to deep neural networks. It helps to understand state-of-the-art development more comprehensively.

3. A fair comparison among models is made by covering BraTS benchmark dataset. The methods are classified, their parameters tabulated and analyzed for performance.

4. The paper also covers a survey on end to end methods for brain tumor segmentation and overall survival prediction. It helps to understand the impact of segmentation on overall survival prediction.

The flow of the article is as follows: section 2 covers the challenges for computer-aided medical image analysis. Section 3 covers the problem statement, dataset, and evaluation framework. In contrast, section 4 includes segmentation methods using hand-crafted features with limitations, section 5 covers segmentation and OS prediction using CNN methods, section 6 covers the limitations of tumor segmentation and OS prediction methods followed by a conclusion and discussion in section 7 .

\section{Challenges in Medical Image Analysis}

Volumetric brain MRI images are analyzed and interpreted by human experts (neurologists, radiologists) to segment various brain tissues as well as to locate the tumor. This analysis is time-consuming. Besides, this type of segmentation is non-reproducible. Accuracy of brain tumor segmentation, which is desirable to plan proper treatment like medication or surgery, highly depends on the human expert with utmost precision. The computer-aided analysis helps a human expert to locate the tumor in less time as well as it regenerates the analysis results. The intended analysis by computerized methods requires appropriate input with correct working methods. Input to the method may face the following challenges:

1. Low signal to noise ratio (SNR) and artifacts in raw MRI data are mainly due to electronic interferences in the receiver circuits, radiofrequency emissions due to thermal motion of the ions in the patient body, coils, and electronic circuits in MRI scan- ners. This random fluctuation reduces the image contrasts due to signal-dependent data bias[49].

2. Non-uniformity is an irrelevant additional intensity variation throughout the MRI signal. Possible causes of non-uniformity are radio-frequency coils, acquisition pulse sequence, the geometry and nature of the sample.

3. The unwanted information acquired by the MR machines along with the brain images like skull, fat, and skin.

4. The intensity profile of MR images may vary due to the variety of MRI machine configuration.

5. Publicly available brain tumor images for computeraided analysis are very less. The collection of MR images from various hospitals has privacy or confidentiality related issues.

6. The class imbalance problem is another major issue in medical image analysis. The images for the abnormal class might be challenging to find because the abnormal classes are rare compared to the normal classes.

\section{Glioma Brain Tumor Segmentation}

The focus on the methods to solve medical related issues has increased since the late 1990s, which is apparent by looking at the gradual increase in semi-automated or automated methods for tumor segmentation, as shown in Fig. 3. By considering the same, the main focus of the article is on the Glioma brain tumor segmentation. An additional task is about the survival prediction techniques of the patients suffering from Glioma.

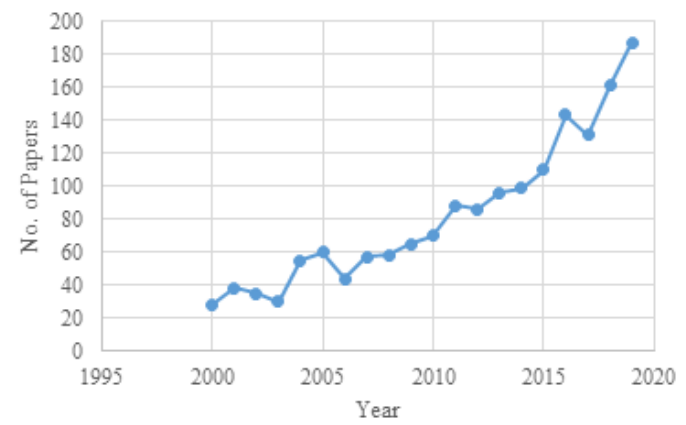

Fig. 3: Papers on PubMed with keywords 'Brain Tumor Segmentation'. 


\subsection{Brain Tumor Segmentation(BraTS) Challenge} Dataset

The literature proposes various methods for brain tumor segmentation. All the methods claim its superiority and usefulness in some way. Initially, all such methods work on the images taken from some hospitals or the radiology laboratories, which are private, and disclosure of those images to the other researchers was not allowed. It did not allow comparison of different methods. The publicaly available dataset and evaluation framework $^{1}$ compares and evaluates the methods on the same measure. The BraTS challenge dataset[13],[11],[12] has the following characteristics:

- It contains multi-parametric MRI pre and post operative scans in T1, T1Gd, T2, and T2-FLAIR volumes(The post operative scans were omitted since 2014);

- The dataset contains images with different clinical protocols $(2 \mathrm{D}$ or $3 \mathrm{D})$ and various scanners from multiple institutions $(1.5 \mathrm{~T}$ or $3 \mathrm{~T})$;

- The dataset set includes the images with preprocessing for their harmonization and standardization without affecting the apparent image information;

- It has co-registration to the same anatomical template, interpolation to a uniform isotropic resolution $\left(1 \mathrm{~mm}^{3}\right)$, and skull-stripping.

Initially, the clinical images in the dataset were very less and it was challenging to compare methods based on the results on such a small number of images. The comparison is possible with increase in number of sample images and accurate generation of the ground truth images. The ground truth is generated based on the evaluation by more than one expert to avoid inter-observer variability. The growth of the dataset from its inception is as shown in Table 3 .

Four different types of intra-tumoral structures are useful for ground truth generation: edema, enhancing core, non-enhancing(solid) core, and necrotic(or fluidfilled) core as shown in Fig 4. An annotation protocol was used by expert raters to annotate each case manually. Then the segmentation results from all raters were fused to obtain a single unanimity segmentation for each subject as the ground truth. The validation of the segmentation methods is based: 1) Whole Tumor (WT): all intra-tumoral substructures, 2) Tumor Core(TC): enhancing tumor, necrosis, and non-enhancing tumor substructures and 3) Enhancing Tumor(ET): includes only enhancing substructure.

1 IPP - ipp.cbica.upenn.edu

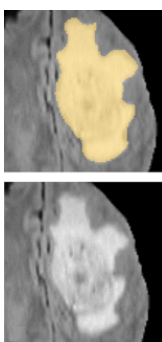

(a)

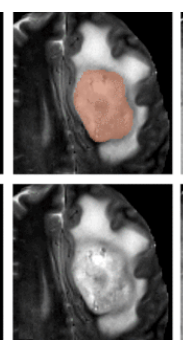

(b)

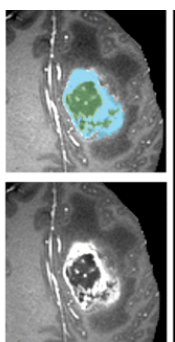

(c)

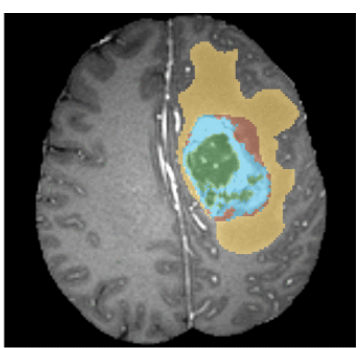

(d)
Fig. 4: Intra-tumoral structures appearance on three imaging modalities with manual annotations. (a) Top: whole tumor (yellow), Bottom:FLAIR, (b) Top: tumor core (red), Bottom:T2, (c) Top: enhancing tumor structures (light blue), surrounding the cystic/necrotic components of the core (green), bottom:T1c, (d) Fusion of the three labels [100].

\subsubsection{Overall Survival Prediction}

On account of sufficient dataset availability, the additional task of overall survival(OS) prediction is introduced in BraTS Challenge since 2017. This task focuses on the OS prediction of HGG patients. The dataset includes age, survival days, and resection status: Gross Total Resection (GTR) or Sub-Total Resection (STR) information for HGG patients in addition to the images. The task is to classify the patients in: long-survivors $(>15$ months), mid-survivors(between 10 to 15 months), and short-survivors ( $<10$ months). The detailed description of OS prediction task is given in Table 4. In 2019, the additional task included the quantification of uncertainty prediction in segmentation. This task focuses on the uncertainty prediction in the context of glioma tumor segmentation.

\subsection{Evaluation Metrics for Brain Tumour}

Segmentation and OS prediction

The standard evaluation framework for tumor segmentation and OS prediction includes the following metrics.

1. Dice Similarity Coefficient (DSC) (or F1 measure): It is the overlap of two objects divided by the total size of both the objects. True Positive(TP) is the outcome where the model correctly predicts the positive class. In contrast, False Positive(FP) is the outcome where the model incorrectly predicts the negative class to be positive. False Negative(FN) is the outcome where the model incorrectly predicts the positive class to be negative.

$$
D S C=\frac{2 T P}{2 T P+F P+F N}
$$


Table 3: Growth of the BraTS dataset[13],[11],[12].

\begin{tabular}{|c|c|c|c|c|c|c|}
\hline Year & $\begin{array}{l}\text { Total Im- } \\
\text { ages }\end{array}$ & $\begin{array}{l}\text { Training Im- } \\
\text { ages }\end{array}$ & $\begin{array}{l}\text { Validation } \\
\text { Images }\end{array}$ & Test Images & Tasks & Type of Data \\
\hline 2012 & $\begin{array}{l}\text { Clinical: } 45 \text {, } \\
\text { Syn- } \\
\text { thetic: } 65\end{array}$ & $\begin{array}{l}\text { Clinical data: } \\
30(20 \mathrm{HGG}+ \\
\text { 10LGG }) \text {, Syn- } \\
\text { thetic data: } \\
50(25 \mathrm{HGG}+ \\
25 \mathrm{LGG})\end{array}$ & $\mathrm{N} / \mathrm{A}$ & $\begin{array}{l}\text { Clinical data: } 15 \text { Syn- } \\
\text { thetic data: } 15\end{array}$ & Segmentation & $\begin{array}{l}\text { Pre and post opera- } \\
\text { tive scans }\end{array}$ \\
\hline 2013 & 65 & $\begin{array}{l}\text { Clinical data } \\
\text { from BraTS } \\
2012\end{array}$ & $\mathrm{~N} / \mathrm{A}$ & $\begin{array}{l}\text { Leaderboard: } \\
\text { Clinical:25 (15 from } \\
\text { BraTS 2012 + } 10 \\
\text { new) } \\
\text { Challenge: } 10\end{array}$ & Segmentation & $\begin{array}{l}\text { Pre and post opera- } \\
\text { tive scans }\end{array}$ \\
\hline 2014 & 238 & 200 & $\mathrm{~N} / \mathrm{A}$ & 38 & $\begin{array}{l}\text { Segmentation, } \\
\text { Disease Pro- } \\
\text { gression }\end{array}$ & $\begin{array}{l}\text { Pre-operative, Longi- } \\
\text { tudinal }\end{array}$ \\
\hline 2015 & 253 & 200 & $\mathrm{~N} / \mathrm{A}$ & 53 & $\begin{array}{l}\text { Segmentation, } \\
\text { Disease Pro- } \\
\text { gression }\end{array}$ & $\begin{array}{l}\text { Pre-operative, Longi- } \\
\text { tudinal }\end{array}$ \\
\hline 2016 & 391 & 200 & $\mathrm{~N} / \mathrm{A}$ & 191 & $\begin{array}{l}\text { Segmentation, } \\
\text { Disease Pro- } \\
\text { gression }\end{array}$ & $\begin{array}{l}\text { Pre-operative, Longi- } \\
\text { tudinal }\end{array}$ \\
\hline 2017 & 477 & 285 & 46 & 146 & $\begin{array}{l}\text { Segmentation, } \\
\text { Survival Pre- } \\
\text { diction }\end{array}$ & $\begin{array}{l}\text { Pre-operative, Longi- } \\
\text { tudinal }\end{array}$ \\
\hline 2018 & 542 & 285 & 66 & 191 & $\begin{array}{l}\text { Segmentation, } \\
\text { Survival Pre- } \\
\text { diction }\end{array}$ & $\begin{array}{l}\text { Pre-operative, Longi- } \\
\text { tudinal }\end{array}$ \\
\hline 2019 & 626 & 335 & 125 & 166 & $\begin{array}{l}\text { Segmentation, } \\
\text { Survival } \\
\text { Prediction, } \\
\text { Uncertainty } \\
\text { Prediction }\end{array}$ & $\begin{array}{l}\text { Pre-operative, Longi- } \\
\text { tudinal }\end{array}$ \\
\hline
\end{tabular}

Table 4: The distribution of BraTS dataset[13],[11],[12] features in survival classes.

\begin{tabular}{|c|c|c|c|c|c|c|c|c|c|c|c|}
\hline \multirow[t]{2}{*}{ Year } & \multirow[t]{2}{*}{ \# records } & \multirow[t]{2}{*}{ Features } & \multicolumn{3}{|c|}{$\begin{array}{l}\text { Short survivors } \\
\text { (<10 months })\end{array}$} & \multicolumn{3}{|c|}{$\begin{array}{c}\text { Long survivors } \\
\text { (between } 10 \text { and } 15 \\
\text { months) }\end{array}$} & \multicolumn{3}{|c|}{$\begin{array}{l}\text { Long Survivors } \\
\text { (>15 months) }\end{array}$} \\
\hline & & & count & $\begin{array}{l}\text { Age }(\mu \pm \\
\sigma)\end{array}$ & $\begin{array}{l}\text { OS days } \\
(\mu \pm \sigma)\end{array}$ & count & $\begin{array}{l}\text { Age }(\mu \pm \\
\sigma)\end{array}$ & $\begin{array}{l}\text { OS days } \\
(\mu \pm \sigma)\end{array}$ & count & $\begin{array}{l}\text { Age }(\mu \pm \\
\sigma)\end{array}$ & $\begin{array}{l}\text { OS days } \\
(\mu \pm \sigma)\end{array}$ \\
\hline 2017 & 163 & Age & 65 & $\begin{array}{l}65.44 \\
10.68\end{array}$ & $\begin{array}{l}147.44 \pm \\
83.08\end{array}$ & 50 & $\begin{array}{l}58.70 \\
11.26\end{array}$ & $\begin{array}{l}394 \\
49.32\end{array}$ & 48 & $\begin{array}{l}55.11 \\
12.19\end{array}$ & $\begin{array}{l}826.23 \pm \\
370.91\end{array}$ \\
\hline 2017 & 163 & Age & 65 & $\begin{array}{l}65.44 \\
10.68\end{array}$ & $\begin{array}{l}147.44 \pm \\
83.08\end{array}$ & 50 & $\begin{array}{l}58.70 \\
11.26\end{array}$ & $\begin{array}{l}394 \\
49.32\end{array}$ & 48 & $\begin{array}{l}55.11 \\
12.19\end{array}$ & $\begin{array}{l}826.23 \pm \\
370.91\end{array}$ \\
\hline 2019 & 212 & $\begin{array}{l}\text { Age, } \\
\text { Resec- } \\
\text { tion } \\
\text { status }\end{array}$ & 82 & $\begin{array}{l}66.66 \pm \\
11.42\end{array}$ & $\begin{array}{l}150.21 \pm \\
84.72\end{array}$ & 54 & $\begin{array}{l}59.14 \pm \\
10.98\end{array}$ & $\begin{array}{l}377.43 \pm \\
40.44\end{array}$ & 76 & $\begin{array}{l}57.16 \pm \\
11.84\end{array}$ & $\begin{array}{l}796.38 \pm \\
354.32\end{array}$ \\
\hline
\end{tabular}


2. Jaccard Similarity Coefficient: It is known as the intersection over the union of two different sets.

Jaccard $=\frac{T P}{T P+F P+F N}$

3. Sensitivity: It is a measure that correctly identifies tumorous voxels.

Sensitivity $=\frac{T P}{T P+F N}$

4. Hausdorff distance: It measures how far two subsets of a metric space are from each other. If $x$ and $y$ be two non-empty subsets of a metric space (M,d), then their Hausdorff distance $d_{H}(x, y)$ can be defined by

$$
d_{H}(x, y)=\max \left\{\sup _{x \in X} \inf _{y \in Y} d(x, y), \sup _{y \in Y} \inf _{x \in X} d(x, y)\right\}
$$

Where sup represents the supremum and inf the infimum.

5. The OS prediction is measurable with accuracy, which is defined to be the quality of being precise.

As DSC is the most commonly used evaluation metric, this article compares all the methods based using DSC unless specified explicitly.

\subsection{Image Pre-processing}

Medical image preprocessing plays a significant role in the appropriate input to the computer-assisted analysis techniques. The preprocessed images help to get the accurate outputs as such images show proper voxel relationships. As mentioned in section 3.1, the dataset has images with different clinical protocols and scanners; the variability is to standardize, and it is necessary to put all the scans on a single scale. In addition to the image registration, uniform isotropic resolution, and skullstripping, following preprocessing further improve the image input:

- Bias Field Correction(BFC): Bias field is a multiplicative field added in the image due to the magnetic field and radio signal in the MR machine. Authors in [139] have suggested a bias field correction technique.

- Intensity Normalization(IN): Different modality images have a separate intensity scale. They must map to the same range. Standardization of all the scans considers zero mean and unit variance.

- Histogram Matching(HM): Due to the different configuration of MR machines, the intensity profile of the acquired images may vary. Intensity profiles are to be brought to the same scale using the histogram matching process.
- Noise Removal(NR): Noise in MR image at the time of acquisition is due to the radio signal, or the magnetic field. Various noise filtering techniques are useful for the removal of noise.

\subsection{Image Post-processing}

The segmentation output generated by computer-assisted methods may contain false segmentation in the image due to improper or incorrect feature selection. The segmentation improves by applying post-processing techniques like:

1. Connected Component Analysis (CCA): CCA groups the voxels based on the connectivity depending on the similar voxel intensities values. The connected components which are very small are excluded from the result as such components are considered to be false positives due to spurious segmentation results.

2. Conditional Random Field(CRF): The classifier predicts the voxel class based on the features related to that voxel, which does not depend on the neighbouring relationship of that voxel with other nearby voxels. CRF takes this relationship into consideration and builds a graphical model to implement the dependencies between the predictions.

3. Morphological Operations: Such operations are applied to adjust the voxel value based on the value of the other voxels in its neighborhood according to the size and shape.

\section{Segmentation Methods using Handcrafted Features}

The methods in this section are divided into two categories: interactive and non-interactive. The interactive methods require user input in form of tumor diameter, boundary or seed point selection. Random field based methods belong to this category. Non-interactive methods do not require user input. First group of this category detects abnormality. In atlas based methods, the abnormal image is non-linearly mapped to the normal/input specific atlas to identify the abnormality which in general is the area of the image where the atlas mapping fails. The other approach is Expectation Maximisation(EM), where the normal and abnormal voxels intensity distribution is learnt with Gaussian Mixture Models(GMM) or probabilistic atlases. The second group of this category is machine learning approaches. The clustering approach groups the voxels in numbers of clusters such that one of these clusters will result in tumorous voxels group. In random forest $(\mathrm{RF})$ and neural network(NN) approaches, high dimensional features 
of the images are given for training. The trained model later classifies the unseen voxels. The detailed description of all these approaches is covered in the following sub-sections.

\subsection{Random Field Based Methods}

Authors in [52] took user input for the largest possible tumor diameter from HGG images to find Volume of Interest(VoI) for tumor and background from T1C MRI images, followed by Cellular Automata(CA) to obtain the probability maps for both the regions. The level set surface was further applied to those probability maps to get the final probability maps. They had further extended their approach in [51], which considered multimodal images (T1C and FLAIR images) to segment tumor, edema as well as LGG images as well.

The semi-automatic method in [50] took the rough tumor region boundary as user input and fine-tuned it with the global and local active contour-based model. The tumor region breaks into sub-regions with adaptive thresholding based on a statistical analysis of the intensities of various tumor regions to separate edema from active tumor core. The process repeats for all the slices of a patient. In [35], the main contribution was to incorporate soft model assignments into the calculations of model-free affinities, which were then integrated with model-aware affinities for multilevel segmentation by weighted aggregation algorithm.

In [145], Random Walk(RW) based interactive as well as an iterative method was applied to fine-tune the tumor boundary. RW was applied as an edge-weighted graph in the discrete feature space based on the variation of the distribution density of the voxels in the feature space. The user made an initial tumor seed selection for tumors as well as edema. Afterward, RW was applied to feature space as well as on the image. If the user did not approve the results, then the segmentation process is reinitiated.

In [42], Hidden Markov Random Field (HMRF) based model was used with modified Pott's Model to panelized the neighboring pixels belonging to different classes. In [137], various modality intensity images along with its neighborhood voxel intensities fed into the mapreduce Hidden Markov Model (HMM), and the model was corrected iteratively based on the class labels.

In [131], Gabor filter bank based Bayesian classification follows by MRF classification. Initially, each voxel divides into its constituent class by applying the Gabor filter bank to the input vector (made up of intensities of four modalities at a voxel) to classify the voxel in five different classes (GM, WM, CSF, tumor, and edema).
Next, MRF based classifier applies to the tumor as well as edema classes. It uses voxel intensity and spatial intensity differences over the neighbor voxels. The [38], uses the Non-Negative Matrix to find voxel clusters, which shows the tumor and level set methods to finetune the region boundary.

\subsection{Atlas Based Methods}

Authors in [112], initially identified the abnormal brain tissues by registering the tumorous brain image with healthy tissues brain atlas. This step was followed by identifying the presence of edema using T2 images, and finally, geometric and spatially constraints were applied to detect the tumor and edema regions. Authors in [36] applied an affine transformation to the atlas image to globally match the patient. The lesion was segmented using the Adaptive Template Moderated Spatially Varying statistical Classification (ATM SVC) algorithm. This atlas was then manually seeded by an expert with a single voxel placed on the estimated origin of the patient's lesion, which was followed by the nonlinear demons registration algorithm with the model of lesion growth to deform the seeded atlas to match the patient. Four volumes of the contrast-enhanced agent with meningioma implement the model.

The paper [78] applied the semi-automatic method, which required user input to give the seed point for tumor, radius for each tumor, and seed point for each regular tissue class. The random walk generates tumor priors using initial tumor seeds. The patient-specific atlas was modified to accommodate tumor classes, using a tumor growth model. Empirical Bayes model used the EM framework to update the posterior of tumor, growth model parameters as well as a patient-specific atlas. This work was then extended in [10], where instead of single seed point for various labels, multiple seed points were taken into consideration to find the intensity mean and variance of a specific label. This work focused on preoperative MRI scans. Whereas, it was further extended in [148] to include post-operative scans along with additional features to the GMM. Here the need for manual selection of seed point was also omitted.

\subsection{Expectation Maximisation Based Methods}

The [99], uses spatially varying probability prior (atlas) to label healthy tissues in the brain. The latent probabilistic atlas 'alpha' finds the probability of having a tumor at that voxel. Gaussian distribution for healthy tissue classes, as well as tumorous tissue class, 
was taken into consideration to identify the tumorous tissues in the image by expectation maximization. For the spatial tumor regularization, latent atlas alpha used MRF using channel-specific regularization parameters. In contrast, authors in [118] used probability priors to predict voxel probability using the EM algorithm, followed by the level set framework with a gradient descent approach for parameter estimation.

The authors in [149], applied Gaussian Mixture Model with EM to divide the data into five probable classes. Afterward, the snake method finds the subtle boundary between the tumor and edema based on the snake method. In [138], the Bayesian intensity tissue model uses the expectation-maximization problem using a trimme likelihood estimator (TLE), which was robust against the outliers. This step was followed by a graph cut algorithm to differentiate between tumorous tissues as well as false positives.

The [150], selects super voxels using Simple Linear Iterative Clustering (SLIC) 3D based on the color and proximity of the voxels. Graph cut on MRF was implemented for initial segmentation, followed by histogram construction, histogram matching, and likelihood estimation applied to predict the probability of voxel. In [110], the Posterior probability using the Bayes theorem was calculated, which was followed by Super voxel partitioning using the SLIC algorithm.

\subsection{Clustering Based Methods}

In [34], from all the images, D patches of size $3 \times 3 \times 3$ are selected in the database. A single patch of this small cube uses all the four modalities. Once the database is ready, from the test dataset, the same size patches are extracted and mapped with the database patches with k-nearest neighbors where $k=5$. These five nearest neighbors generate the test patch label.

The [32], uses the knowledge-based multispectral analysis on the top of the unsupervised clustering technique. The rule-based expert system then extracted the intracranial region. The training was performed on three T1, Proton Density(PD), and T2 images, whereas the test on thirteen such volumes. The [122], uses the roughset approximation to fine-tune the prediction done by k-means clustering.

In [116], initially, the enhanced probabilistic fuzzy $\mathrm{C}$-means clustering was applied to get the rough estimation of the tumor region. This estimation and cluster centroid is given to the gradient-vector-flow snake model to refine the tumor boundary. The [126], learns a Sparse dictionary of size $4 \mathrm{x} 4$ for different tissue types using four image modalities followed by logistic regres- sion for the tissue classification. This initial stage classifies the image voxels in various classes. This step was followed by k-means clustering, which uses a very high dimensional feature vector as input to find the classification of the tumor as well as the edema region as an overlap of the output of the previous step.

\subsection{Random Forest Based methods}

The [95] implements a generative- discriminative model. The generative model estimates tissue probability using density forest (similar to GMM). The discriminative model implements classification forest, which took as an input 51 features (gradient information first-order texture features and symmetry-based features, prior tissue probabilities based on the density forest) to generate the probability of the tissue. This probability was then supplied to the CRF to fine-tune the result.

Authors in [155] use context-aware spatial features, along with the tissue appearance probability generated by Gaussian Mixture Model, to train decision forest. The [17], works on Random forest classification with CRF regularization to predict the probability of tissue in the multiple class, i.e., GM, WM, CSF, Edema, Necrotic core and enhancing tumor. 28-dimensional feature vector (includes the intensity of each modality along with first-order statistics like mean, variance, skewness, kurtosis, energy, and entropy computed from local patches around each voxel in each modality.

In [47], each voxel was characterized by signal modality as well as spatial prior. The averaging across a $3 \times 3 \times 3$ cube removes the noise. The random forest trains using local features (intensity or priors), as well as contextspecific features (region-based features or symmetrybased features). The forest was made up of 30 trees with a depth of 20 for each tree and trained on the synthetic data.

The [156], uses a discriminative multiclass classification forest. It uses spatially non-local context-sensitive high dimensional features along with the prior probability for the tissue to be classified. Prior probability is available to the GMM. It classifies in three classes, i.e., background, edema, and tumor. Three types of features train 40 tree with a depth of 20 each, and such features were intensity difference, intensity mean difference, and intensity range on a 3D line to check for structural changes. 2000 combination was selected to design the decision trees. The [46], trains the random forest of 50 trees each of depth 25 on 120000 samples with 324 features comprises of intensity, neighborhood information, context information, and texture information. 
In [119], Random Forest classifier used intensities, the difference of intensities (T1 - T2, T1- Flair, T1 - T1c), and the texture-based features such as fractal and texton to train RF using three-fold cross-validation. They further extended their work in [120] for the postprocessing, where the connected component analysis removes tiny regions in the $3 \mathrm{D}$ volume, and holes in between the according to the neighboring region.

In [48], Extremely Randomized Trees(ExtraTrees) were used, which introduces more randomness at the time of training. The classifier was trained on 208 features extracted from all the four modalities, which include intensity values, local histograms, first-order statistics, second-order statistics, and basic histogram-based segmentation. In the paper, the ExtraTrees trains with the best threshold rather than the threshold derived from the individual features. In [75], pixel classification was done with ten random forests with ten trees each, which are trained in parallel to reduce the training time and finally merged into a single forest with Gini impurity. One thousand samples for tumorous class and 1000 samples for the non-tumorous class trains the RF. Classification forest in [96] used 237 features which included appearance specific features (image intensities, first-order texture features, and gradient features), context sensitive features (ray feature, symmetry intensity difference features). Authors in [88], [97], [82], [98], [43], [129], [80], [109] had used random forest classifier with combination of various intensity based features, gradient based features, texture based features, rotation invariant features.

In [19], tensor features were extracted along with mean, entropy and standard deviation features. Authors in [123] extracted features for supervoxels from multi scale images and created sparse feature vectors to segment whole tumor. Sub-regions of tumor were then separated using CRF.

\subsection{Neural Network Based methods}

In [20], Grouping Artificial Immune Network(GAIN) takes as an input voxel intensity from $2 \mathrm{D}$ as well as $3 \mathrm{D}$ slices, statistical features and texture features for training as well as segmentation of brain MRI images. Information is in the form of bits from various image modalities. In [2], Convolutional Restricted Boltzmann Machine trains GMM and spatial tissue priors.

Table 5 summarizes the methods for the type of pre-preprocessing, dataset, the number of images used as well as the DSC achieved. The DSC is shown for the various tumor sub-regions which includes WT, ET, TC and Edema(ED) for training, validation and test datasets.
The DSC comparison of different methods for tumor segmentation is shown in Fig. 5. The atlas and RFbased methods performed well compared to all other approaches.

Random field methods, atlas-based methods, expectation maximization methods, and clustering methods do not use any post-processing techniques. Random forest based methods, authors in [46], [120] and [75] used connected component analysis, [96], [97], [129] applied spatial regularization and authors in [88] and [43] applied morphological operations to refine the segmentation output.

Table 5: Summarization of segmentation methods using handcrafted features.

\begin{tabular}{|c|c|c|c|c|}
\hline Ref. & $\begin{array}{l}\text { Pre- } \\
\text { processing }\end{array}$ & Dataset & $\begin{array}{l}\# \text { im- } \\
\text { ages }\end{array}$ & DSC Mean \\
\hline \multicolumn{5}{|c|}{ Random Field methods } \\
\hline$[52]$ & - & Custom & 29 & $\begin{array}{l}\text { Training } \\
\text { TC:0.89 } \\
\text { Test } \\
\text { TC:0.80 }\end{array}$ \\
\hline$[51]$ & - & $\begin{array}{l}\text { BraTS } \\
2012\end{array}$ & 30 & TC:0.69,ED:0.37 \\
\hline$[50]$ & - & $\begin{array}{l}\text { BraTS } \\
2013\end{array}$ & 30 & TC:0.82 \\
\hline$[35]$ & IN & Custom & 20 & $\begin{array}{l}\text { Training } \\
\text { TC:0.70,ED:0.66 } \\
\text { Test } \\
\text { TC:0.66,ED:0.61 }\end{array}$ \\
\hline$[145]$ & $\mathrm{NR}$ & $\begin{array}{l}\text { BraTS } \\
2012\end{array}$ & 30 & TC:0.53,ED:0.25 \\
\hline$[42]$ & - & $\begin{array}{l}\text { BraTS } \\
2013\end{array}$ & 30 & $\begin{array}{l}\text { HGG } \\
\text { WT:0.84,TC:0.54, } \\
\text { ET:0.67 } \\
\text { LGG } \\
\text { WT:0.81,TC:0.54, } \\
\text { ET:0.11 }\end{array}$ \\
\hline$[131]$ & - & $\begin{array}{l}\text { BraTS } \\
2012\end{array}$ & 28 & TC:0.66,ED:0.56 \\
\hline$[137]$ & $\mathrm{BFC}$ & $\begin{array}{l}\text { BraTS } \\
2013\end{array}$ & 30 & $\begin{array}{l}\mathrm{HG} \\
\mathrm{TC}: 0.62, \mathrm{ED}: 0.59\end{array}$ \\
\hline \multicolumn{5}{|c|}{ Atlas Based Methods } \\
\hline$[78]$ & $\mathrm{BFC}, \mathrm{IN}$ & $\begin{array}{l}\text { BraTS } \\
2014\end{array}$ & 200 & $\begin{array}{l}\text { Validation } \\
\text { WT:0.86,TC:0.79, } \\
\text { ET:0.59 } \\
\text { Test } \\
\text { WT:0.88,TC:0.83, } \\
\text { ET:0.72 }\end{array}$ \\
\hline
\end{tabular}


Table5 - continued ...

\begin{tabular}{|c|c|c|c|c|}
\hline Ref. & $\begin{array}{l}\text { Pre- } \\
\text { processing }\end{array}$ & Dataset & $\begin{array}{l}\# \text { im- } \\
\text { ages }\end{array}$ & DSC Mean \\
\hline [10] & $\mathrm{BFC}, \mathrm{IN}$ & $\begin{array}{l}\text { BraTS } \\
2015\end{array}$ & 186 & $\begin{array}{l}\text { Training } \\
\text { WT:0.88,TC:0.77, } \\
\text { ET:0.68 }\end{array}$ \\
\hline [148] & $\mathrm{NR}, \mathrm{HM}$ & $\begin{array}{l}\text { BraTS } \\
2016\end{array}$ & 200 & $\begin{array}{l}\text { WT:0.89,TC:0.77, } \\
\text { ET:0.67 }\end{array}$ \\
\hline \multicolumn{5}{|c|}{ Expectation Maximisation Based Methods } \\
\hline [99] & - & $\begin{array}{l}\text { BraTS } \\
2012\end{array}$ & 30 & $\begin{array}{l}\text { HGG } \\
\text { TC:0.55,ED:0.57 } \\
\text { LGG } \\
\text { TC:0.24,ED:0.42 }\end{array}$ \\
\hline$[118]$ & - & $\begin{array}{l}\text { BraTS } \\
2012\end{array}$ & 30 & $\begin{array}{l}\text { HGG } \\
\text { TC:0.58,ED:0.60 } \\
\text { LGG } \\
\text { TC:0.32,ED:0.36 }\end{array}$ \\
\hline [149] & - & $\begin{array}{l}\text { BraTS } \\
2012\end{array}$ & 30 & TC:0.31,ED:0.35 \\
\hline [138] & - & $\begin{array}{l}\text { BraTS } \\
2012\end{array}$ & 30 & TC:0.43,ED:0.55 \\
\hline$[150]$ & IN & $\begin{array}{l}\text { BraTS } \\
2013\end{array}$ & 30 & $\begin{array}{l}\text { HGG } \\
\text { WT:0.83,TC:0.74, } \\
\text { ET:0.68 } \\
\text { LGG } \\
\text { WT:0.83,TC:0.58, } \\
\text { ET:0.51 }\end{array}$ \\
\hline [110] & - & $\begin{array}{l}\text { BraTS } \\
2015\end{array}$ & 200 & $\begin{array}{l}\text { Training } \\
\text { WT:0.74,TC:0.55, } \\
\text { ET:0.54 }\end{array}$ \\
\hline \multicolumn{5}{|c|}{ Clustering Based Methods } \\
\hline$[34]$ & - & $\begin{array}{l}\text { BraTS } \\
2013\end{array}$ & 30 & $\begin{array}{l}\text { HGG } \\
\text { WT:0.79,TC:0.60, } \\
\text { ET:0.59 } \\
\text { LGG } \\
\text { WT:0.76,TC:0.64, } \\
\text { ET:0.44 }\end{array}$ \\
\hline$[122]$ & - & $\begin{array}{l}\text { BraTS } \\
2013 \\
\text { BraTS } \\
2015\end{array}$ & 200 & $\begin{array}{l}\text { WT:0.82,TC:0.71, } \\
\text { ET:0.72 }\end{array}$ \\
\hline$[116]$ & - & Custom & 15 & TC:0.82 \\
\hline$[126]$ & - & $\begin{array}{l}\text { BraTS } \\
2012\end{array}$ & 30 & TC:0.30,ED:0.39 \\
\hline \multicolumn{5}{|c|}{ Random Forest Based Methods } \\
\hline [95] & - & $\begin{array}{l}\text { BraTS } \\
2013\end{array}$ & 30 & $\begin{array}{l}\text { HGG } \\
\text { WT:0.80,TC:0.69, } \\
\text { ET:0.69 } \\
\text { LGG } \\
\text { WT:0.76,TC:0.58, } \\
\text { ET:0.20 }\end{array}$ \\
\hline$[155]$ & - & Custom & 40 & $\begin{array}{l}\text { TC:0.85,NC:0.75, } \\
\text { ET:0.80 }\end{array}$ \\
\hline$[17]$ & $\begin{array}{l}\text { BFC, IN, } \\
\text { HM }\end{array}$ & $\begin{array}{l}\text { BraTS } \\
2012\end{array}$ & 30 & $\begin{array}{l}\text { HGG-real } \\
\text { TC:0.62,ED:0.61 } \\
\text { LGG-real } \\
\text { TC:0.49,ED:0.35 }\end{array}$ \\
\hline
\end{tabular}

Table5 - continued ...

\begin{tabular}{lllll}
\hline Ref. & $\begin{array}{l}\text { Pre- } \\
\text { processing }\end{array}$ & Dataset & $\begin{array}{l}\text { \# im- } \\
\text { ages }\end{array}$ & DSC Mean \\
\hline$[47]$ & HM & $\begin{array}{l}\text { BraTS } \\
2012\end{array}$ & 30 & $\begin{array}{l}\text { HGG-real } \\
\text { TC:0.68,ED:0.56 } \\
\end{array}$ \\
& & & $\begin{array}{l}\text { LGG-real } \\
\text { TC:0.52,ED:0.29 }\end{array}$ \\
& & & & HGG-real \\
& & BraTS & 30 & TC:0.71,ED:0.70 \\
\hline 156$]$ & BFC & 2012 & & TC:0.62,ED:0.44 \\
& & & & HGG \\
& & & & WT:0.83, TC:0.70, \\
\hline$[46]$ & BFC, HM & BraTS & 30 & ET:0.75
\end{tabular}

ET:0.75

LGG

WT:0.72,TC:0.47, ET:0.21

\begin{tabular}{|c|c|c|c|c|}
\hline$[119]$ & $\mathrm{BFC}, \mathrm{HM}$ & $\begin{array}{l}\text { BraTS } \\
2013\end{array}$ & 30 & $\begin{array}{l}\text { HGG } \\
\text { WT:0.92,TC:0.91, }\end{array}$ \\
\hline
\end{tabular}

ET:0.88

LGG

WT:0.92,TC:0.91, ET:0.88

\begin{tabular}{llll}
\hline$[120]-$ & BraTS 200 & Training \\
2014 & & WT:0.81,TC:0.66, \\
& & ET:0.71
\end{tabular}

\begin{tabular}{llll}
\hline 48$]$ & BFC, HM & BraTS 208 & WT:0.83,TC:0.71, \\
& 2013
\end{tabular}

\begin{tabular}{llll}
\hline [75] HM, IN & $\begin{array}{l}\text { BraTS } \\
2014\end{array}$ & Training \\
& & WT:0.84,TC:0.68,
\end{tabular}

ET:0.72

Valid/Test

WT:0.87,TC:0.76, ET:0.64

\begin{tabular}{llllll}
\hline [96] & NR, & IN, & BraTS & 30 & Training \\
BFC & & 2013 & & WT:0.83,TC:0.66,
\end{tabular}

ET:0.58

Challenge

WT:0.84,TC:0.73, ET:0.68

[88] BFC, IN BraTS $252 \quad$ WT:0.75,TC:0.60,

\begin{tabular}{llll} 
& 2015 & ET:0.56 \\
\hline$[97]-$ BraTS & 65 & WT:0.83,TC:0.69,
\end{tabular}

\begin{tabular}{llll} 
& 2015 & ET:0.63 \\
\hline$[82]$ & BFC, NR, & BraTS 30 & WT:0.87,TC:0.88
\end{tabular}

\begin{tabular}{lllll} 
& IN & 2013 & & \\
\hline$[98]$ & - & BraTS 30 & Valid \\
& 2013 & & WT:0.79, TC:0.75,
\end{tabular}

ET:0.66

Challenge

WT:0.83,TC:0.76, ET:0.71

\begin{tabular}{lllll}
\hline$[43]$ & BFC, HM & BraTS & 200 & WT:0.80,TC:0.72, \\
& & 2016 & & ET:0.73 \\
\hline$[129]$ & IN & BraTS & 274 & Training \\
& & 2015 & & WT:0.87, TC:0.72, \\
& & & ET:0.75 \\
\hline
\end{tabular}


Table5 - continued ...

\begin{tabular}{|c|c|c|c|c|}
\hline Ref. & $\begin{array}{l}\text { Pre- } \\
\text { processing }\end{array}$ & Dataset & $\begin{array}{l}\# \text { im- } \\
\text { ages }\end{array}$ & DSC Mean \\
\hline$[80]$ & IN & $\begin{array}{l}\text { BraTS } \\
2015\end{array}$ & 274 & $\begin{array}{l}\text { Training } \\
\text { WT:0.84,TC:0.7 } \\
\text { ET:0.71 }\end{array}$ \\
\hline$[109]$ & $\mathrm{BFC}$ & $\begin{array}{l}\text { BraTS } \\
2017\end{array}$ & 285 & $\begin{array}{l}\text { Training } \\
\text { WT:0.64,TC:0.4 } \\
\text { ET:0.47 } \\
\text { Test } \\
\text { WT:0.63,TC:0.4 } \\
\text { ET:0.42 }\end{array}$ \\
\hline$[19]$ & IN, HM & $\begin{array}{l}\text { BraTS } \\
2017\end{array}$ & 285 & $\begin{array}{l}\text { Validation } \\
\text { WT:0.79,TC:0.6 } \\
\text { ET:0.61 } \\
\text { Test } \\
\text { WT:0.77,TC:0.6 } \\
\text { ET:0.50 }\end{array}$ \\
\hline$[123]$ & - & $\begin{array}{l}\text { BraTS } \\
2018\end{array}$ & 285 & $\begin{array}{l}\text { Validation } \\
\text { WT:0.80,TC:0.6 } \\
\text { ET:0.57 } \\
\text { Test } \\
\text { WT:0.73,TC:0.5 } \\
\text { ET:0.50 }\end{array}$ \\
\hline \multicolumn{5}{|c|}{ Neural Network Based Methods } \\
\hline$[20]$ & - & $\begin{array}{l}\text { BraTS } \\
2013\end{array}$ & 30 & $\begin{array}{l}\text { WT: } 0.73, \text { TC: } 0.5 \\
\text { ET:0.63 }\end{array}$ \\
\hline$[2]$ & - & $\begin{array}{l}\text { BraTS } \\
2015\end{array}$ & 200 & $\begin{array}{l}\text { Test } \\
\text { WT:0.81, } \\
\text { TC:0.68, } \\
\text { ET:0.65 }\end{array}$ \\
\hline
\end{tabular}

The limitations associated with the methods working on handcrafted features are as follows:

1. Identifying tissue probability classes: tumor tissue intensities overlap with that of the healthy tissues, as mentioned in Table 1; in such a case identifying the probable class for tumorous tissue is quite challenging.

2. Atlas matching (healthy or tumorous atlas): Usually, the brain atlas contains the normal brain tissue distribution map. Due to the deformation of the healthy tissue by the tumor, the atlas matching of a tumorous brain may result in the wrong map

3. Manual seed point identification for the tumor or its subparts: Almost all semi-automated methods require some initial selection for the tumorous voxel, its diameter, or its rough outer boundary. The selection depends on the expert. Its repetition over all the slices of the brain is a time-consuming task.

4. Feature extraction from the images: RF training depends on the features extracted from the brain images. All the MRI modalities contain different biological information. This variation in the information complicates the task of feature extraction as well as selection to training RF.
5. Discontinuity: The results generated by such methods are spurious, which increases the chances of false positives. The proper post-processing techniques are required to fine-tune the generated results.

\section{Deep Neural Network}

Deep Neural Network(DNN) is an artificial intelligence function which mimics the human brain working for data processing and pattern creation in decision making. There are mainly four reasons contributing to their success:

1. The DNN models solved the problem in an end-toend manner. The models learn the features from the data automatically with help of various functions. The feature learning improves from simple features at initial layers to complex features at deeper layers of the model. The automatic feature learning has eliminated the need of domain expertise.

2. The computational capabilities of the hardware in terms of GPU and efficient implementation of the model on GPU with various open source libraries have made the training of the DNN 10 to 50 times faster than CPU.

3. Efficient optimization techniques for robust learning contribute to the success of DNN for optimal network performance.

4. The availability of benchmark datasets allow training and testing of various deep learning models to be implemented successfully.

The exponential growth of the usage of DNN techniques to solve the variety of problems is shown in Fig. 6. A similar growth pattern is identified for solving the brain tumor segmentation problem, which is as shown in Fig. 7.

The general block diagram of any deep learning technique is as shown in Fig 8. The crucial task is to get the labeled data set. After the availability of the dataset, it is divided into training and validation sets, followed by appropriate pre-processing techniques as per the task on hand. Actual DNN applies to the training data, which makes the network learn the network parameters. The output of DNN is spurious at some brain area, and post-processing fine-tunes the segmentation result. And at last, the evaluation framework measures the performance of the network.

\subsection{Evolution of DNN}

In medical image analysis, the semantic segmentation task is common, e.g., segmentation of organ, lesion. 


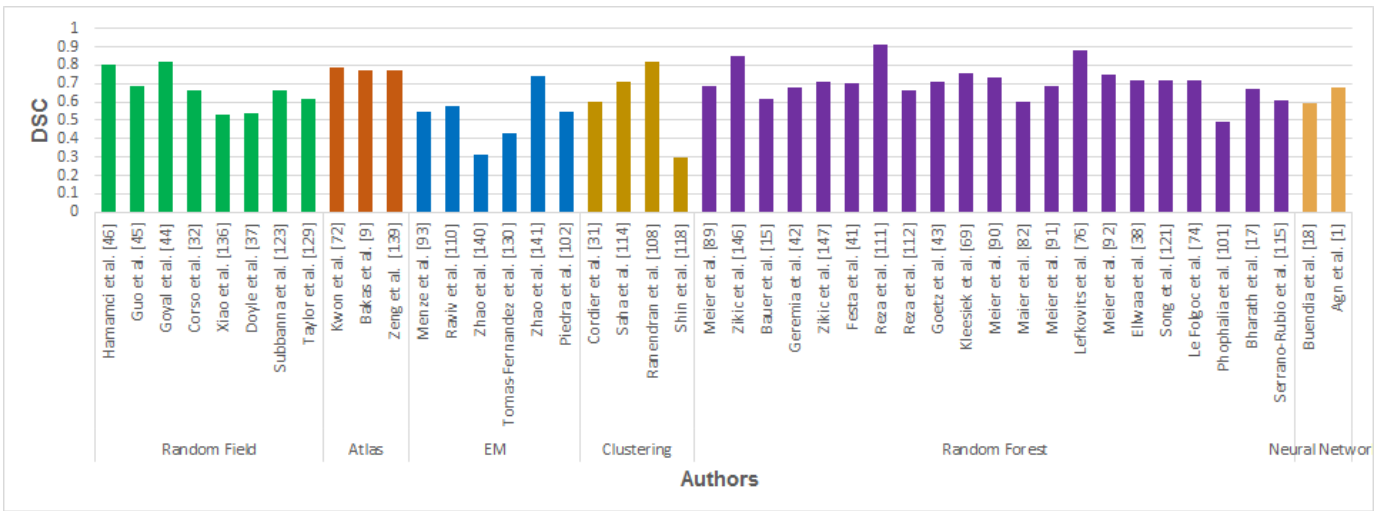

Fig. 5: Comparison of segmentation methods using hand-crafted features (DSC for validation/Test for Tumor/WT of all images/HGG).

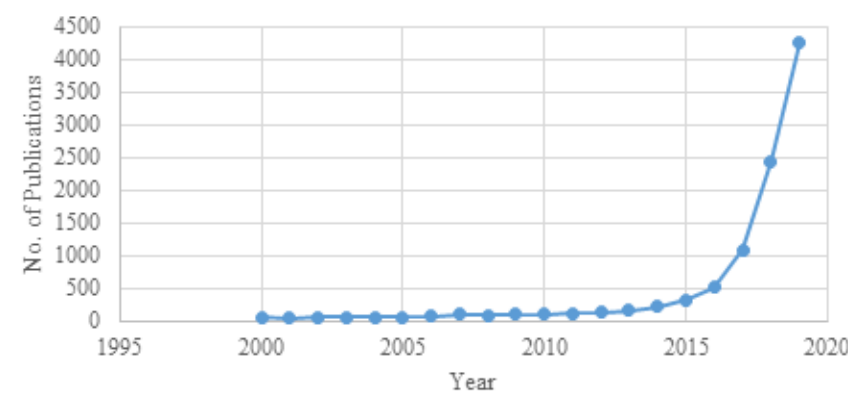

Fig. 6: The growth rate of research papers with the keyword 'deep learning' on PubMed.

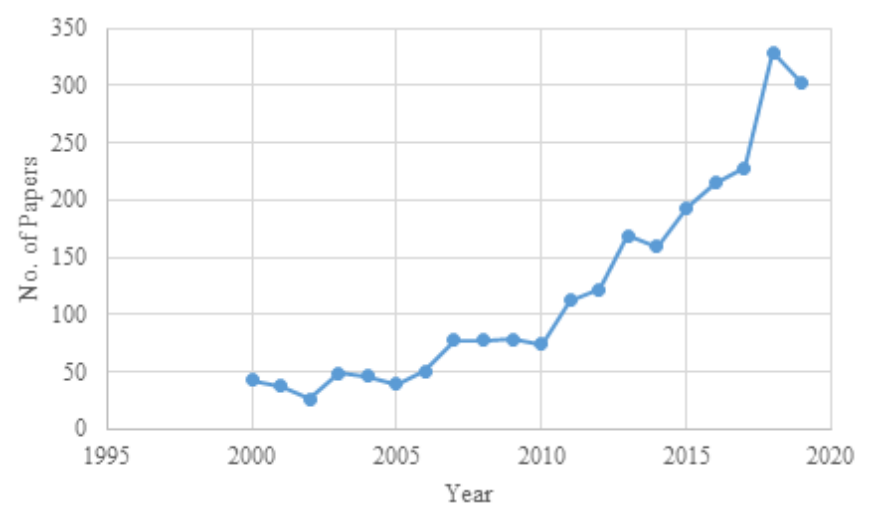

Fig. 7: The growth rate research papers with the keywords 'deep learning' and 'brain tumor segmentation' on PubMed.

The Convolutional Neural Network(CNN), specific type of DNN architecture gained its popularity from 1990 with the architecture of LeNet[81], which was two layers of architecture. After the availability of fast GPUs and other computing facilities, over fifteen years later,
AlexNet was proposed by authors in [77] with five convolutional layers. The CNNs are designed with variety of layers (like convolution layers, non-linearity layers, pooling layers, fully-connected layers), regularization, optimization and normalization, loss functions, as well as network parameters initializations. Authors in [18] have nicely explained the architectural elements of CNN which are as follows:

Convolution layer: extracts representative features from the input. It achieves: 1)weight-sharing mechanism, 2)exploits local connectivity of input, and 3) provides shift invariance at some extent.

Non-linearity layer: provides sparse representation of input space which achieves data variability invariance and computationally efficient representation. Types of non-linearity layers are Rectified Linear Unit(ReLU), Leaky ReLU(LReLU), Parametric ReLU (PReLU), S-shaped ReLU(SReLU), Maxout and its variants, Exponential Linear Unit(ELU) and its variants.

Pooling/subsampling layer: extracts prominent features from non-overlapping neighborhood. It is used to 1 ) reduce no. of parameter, 2) reduce over-fitting, and 3) achieve translation invariance. Commonly used pooling techniques are max pooling and average pooling.

Fully connected layer: converts $2 \mathrm{D}$ features to $1 \mathrm{D}$ feature vector. It helps to predict input image class label.

Loss functions: improves learning process by improving within class similarity and between class separability.

Regularizations: deals with over-fitting issues. Commonly used regularization techniques are L1 and L2 regularizations, dropout, early stopping, batch normalization. 


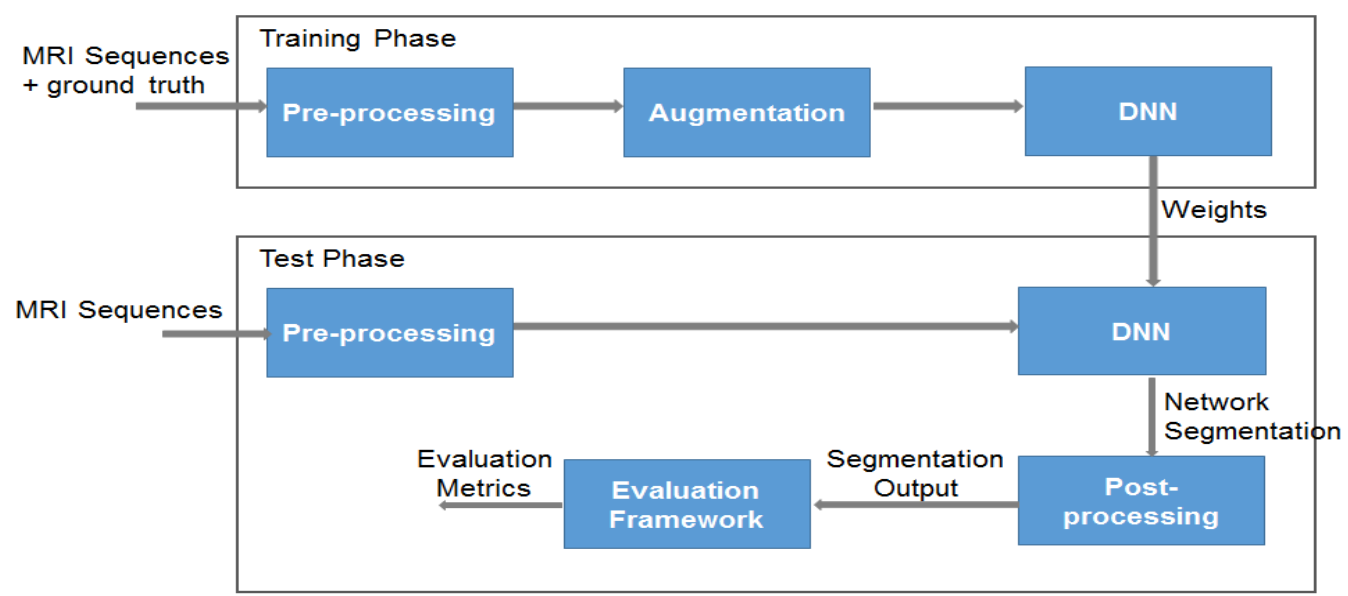

Fig. 8: Generalize DNN network.

Optimization: used for proper updates of network parameters during backpropogation. Various techniques of optimization includes Nesterov accelerated gradient descent, adaptive gradient algorithm (Adagrad), Root Means Square Propogation (RMSProp)

Weight initialization and normalization: boosts the learning process by helping the weight update with proper initial values.

The convolution layers extract features from the input by applying kernels to it. The output feature map depends on the type of kernel and its size. At the initial layers simple features are extracted from the input like edge or lines. The gradual increase of the network depth requires higher number of feature maps to extract complex shapes[5]. The activation function is applied to the feature maps to learn the non-linear relationship within the data and allows the errors to backpropagate to the initial layers for accurate parameter updates. Increase in the network depth exponentially increases the network parameters, which is highly computationally expensive. To balance the growth of the network parameters, pooling layers are introduced to down sample the input feature maps and reduce its spatial size by considering only the prominent features. The fully connected layers at the end of the network flatten the result of the input layers before actual classification. The loss function at the classification layer calculates the error in the prediction. Based on this error, the network parameters are updated using the gradient descent methods by backpropagation. Commonly used loss functions are:

- Cross Entropy loss function:

$$
J=-\frac{1}{N}\left(\sum_{\text {voxels }} y_{\text {true }} \cdot \log \hat{y}_{\text {pred }}\right)
$$

- Dice Loss function:

$$
J=1-\frac{2 \sum_{\text {voxels }} y_{\text {true }} y_{\text {pred }}+\epsilon}{\sum_{\text {voxels }} y_{\text {true }}^{2}+\sum_{\text {voxels }} y_{\text {pred }}^{2}+\epsilon}
$$

Here, $N=$ Number of voxels, $y_{\text {true }}=$ groundtruth label, and $y_{\text {pred }}=$ network predicted label.

The CNNs, with convolution layers followed by fullyconnected layers, classifies entire image in a single category. GoogleNet(Inception)[134] and InceptionV3[135] networks have introduced the inception module which implements the kernels of different sizes to reduce network parameters. ResNet[136] has introduced the residual connection between the convolution layers such that it learns the identity function which allows effective training of deeper networks. In DenseNet[61] layers are very narrow and adds very less number of feature maps to the network which again allows to design deeper architectures and training is efficient as each layer has direct access to the gradient of the loss function.

For semantic segmentation, the CNN can simply be used to classify each voxel of the image individually by presenting it with several patches extracted around the particular voxel. Each voxel of the image is classified with the same process, resulting in the segmentation of the entire image. This 'sliding-window' approach repeats the convolution operations for adjacent patches of neighboring voxels. The improvement to this approach is the replacement of fully-connected layers with convolution layers, which generates the probability map of the entire input image rather than generating output for a single voxel. Such networks are known to be Fully Convolutional Neural Networks(FCNN). FCN[85] is a type of FCNN where skip connections are introduced to reconstruct the high resolution image. U-Net[121], a very known highly adapted network architecture for tumor segmentation has taken the encoder - decoder 
approach where every encoding layer is connected with its peer decoding layer with skip connection to reconstruct the dimension as well to get the particular spatial information from the encoding layer. SegNet [8] and DeepLab[28] are the other type of FCNN architecture adopted to solve the problem of brain tumor segmentation.

\subsection{Handling Class Imbalance Problem}

In medical image analysis, finding the number of abnormal images as compared to the normal images is difficult as abnormality like the tumor is rare. This problem is called 'class imbalance'. All images in this article are of the tumor; the class imbalance issue persists because, in the single brain volume, the proportion of the tumor is less compared to average brain volume. Even on a single brain slice, the area of the tumor is small compared to the brain part, which leads to a class imbalance problem. The proportion of the brain volume (BV) and back ground volume (BGV) with respect to tumor volume for BraTS 2019 dataset is as shown in Table 6 .

Table 6: The proportion of non tumor brain volume (NTBV) and non tumor background volume (NTBGV) vs. tumorous volume(TV).

\begin{tabular}{llccl}
\hline & $\begin{array}{l}\% \\
\text { NTBV }\end{array}$ & \% Necrosis & \% Edema & \% ET \\
\hline BV & 75.75 & 0.7 & 17.78 & 5.77 \\
\hline BGV & 99.11 & 0.03 & 0.65 & 0.21 \\
\hline
\end{tabular}

The following approaches address data imbalance problem.

- Patch sampling: The patch sampling-based method can mitigate the imbalanced data problem. The sampling process includes the equiprobable patches from all the tumorous regions as well as the non-tumorous region.

- Improvement in loss functions: Some of the loss functions, when used in its raw form, may not suit the tumor segmentation task, as they consider balanced datasets. These functions adopt imbalanced dataset with modifications as:

1. The weighted cross-entropy loss function: The voxel-wise class prediction averaged for all voxels may lead to error if the class is imbalanced in the image. The issue is in a weighted crossentropy function where the weight is associated with all the voxels prediction. Since the background regions dominate the training set, it is reasonable to incorporate the weights of multiple classes into the cross-entropy such that more weight given to the voxels of the positive class.

$$
\operatorname{Loss}_{W C E}=\sum_{\text {voxels }} \sum_{\text {classes }} y_{\text {true }} \cdot \log \hat{y}_{\text {pred }}
$$

2. Generalized Dice Loss Function: Authors in [132] proposed to use the class rebalancing properties of the generalized dice. It provides a robust and accurate deep-learning loss function for unbalanced tasks.

$$
\text { Loss }_{G D L}=1-2 \frac{\sum_{\text {classes }} w \sum_{\text {voxels }} y_{\text {true }} y_{\text {pred }}}{\sum_{\text {classes }} w \sum_{\text {voxels }} y_{\text {true }}+y_{\text {pred }}}
$$

3. Focal Loss Function: The detection task uses focal loss. It encourages the model to down-weight easy examples and focuses training on hard negatives. Formally, the focal loss defines a modulating factor to the cross-entropy loss and a parameter for class balancing [84].

$$
\begin{array}{r}
\operatorname{Loss}_{F L}\left(p_{t}\right)=-\alpha_{t}\left(1-p_{t}\right)^{\gamma} \log \left(p_{t}\right) \\
p_{t}= \begin{cases}p_{t} & \text { if } y_{i}=1 \\
1-p_{t} & \text { otherwise }\end{cases}
\end{array}
$$

where $y \epsilon\{1,-1\}$ is the ground-truth class, and $p_{t} \epsilon\{0,-1\}$ is the estimated probability for the class with label $y=1$. The weighting parameter $\alpha$ deals with imbalanced dataset. The focusing parameter $\gamma$ smoothly adjusts the rate at which easy examples are down-weighted. Setting $\gamma>0$ can reduce the relative loss for well-classified examples. It places the focus on hard and misclassified examples; the focal loss is equal to the original cross-entropy loss when $\gamma=0$.

- Augmentation techniques: Most of the time, a large number of labels for training are not available for several reasons. Labeling the dataset requires an expert in this field, which is expensive and timeconsuming. Training large neural networks from limited training data causes the over-fitting problem. Data augmentation is a way to reduce over-fitting and increase the amount of training data. It creates new images by transforming (rotated, translated, scaled, flipped, distorted, and adding some noise such as Gaussian noise) the ones in the training dataset. Both the original image and created images are input to the neural network. For example, a large variety of data augmentation techniques include random rotations, random scaling, random 
elastic deformations, gamma correction augmentation, and mirroring on the fly during training.

\subsection{CNN Methods Classification for Tumor Segmentation}

The classification of CNNs for tumor segmentation uses the combination of the design aspects as shown in the Fig. 9.

Input type: The network may take $2 \mathrm{D} / 3 \mathrm{D}$ input in form of patches or images. The CNN with fullyconnected layers classify the centre voxel of the patch whereas FCNN predicts multiple or all voxels of the patch/image. The network may take multi-scale patches to extract coarse and fine details of the input.

Output Type: The output of the network depends on the problem to solve. It predicts single output for the classification problem and multiple voxel output for the semantic segmentation problem.

Type of network: The CNN approach indicates the convolution network with fully-connected layers at the end whereas FCN indicates the network with all convolution layers.

Ensemble Approach: Ensemble approach can be classified into serial and parallel approaches. In the serial approach multiple networks combine in series to fine tune the end output. The input of one network depends on the output of the other. In the parallel approach multiple networks work in parallel and take the same/different input to gather the comprehensive details from the input. The final output of the network is decided based on the majority voting or averaging of all the network output.

The evolution of CNN based methods for the tumor segmentation is as shown in Fig. 10. Some of well-known CNN architectures for brain tumor segmentation are represented in Fig. 11, Fig. 12, Fig. 13 and Fig. 14.

The architecture of [54] is a two pathway CNN which takes 2D multi-resolution input patches, applies the convolution operations and concatenate the output of both the pathways. The deepmedic[70] also follows two pathways with 3D multi-resolution input patches and incorporates the residual connections and predicts the output for multiple voxels at a time.

U-net[121] is an encoder-decoder architecture with skip-connections between the peer layers of analysis and synthesis path. This architecture has gained most popularity. Anisotropic[142] architecture followed the serial ensemble approach. The first network segments whole tumor, second segments tumor core and considers the output of first network and finally third network segments enhancing tumor with help of second network output.

Initially, shallow CNN performs the voxel-based image segmentation. The authors in [54] proposed voxelwise classification using CNN multipath way architecture. One pathway uses 2D patches of size $32 \times 32$, and the other uses fully-connected input of $5 \times 5$ patch size having center pixel same as $32 \times 32$ patch. Patch selection was made such that the labels are equiprobable. L1 and L2 regularizations overcome overfitting. In [140], voxel-wise class probability prediction uses separate 3D CNNs for HGG and LGG images. The final probability classified the voxel into the six classes. In [157], five layers deep 2D CNN architecture performs voxel-wise classification.

Gradually the depth of CNN had increased to accommodate more layers in the network. In [108], 2D deep CNN with fully connected output layers separates HGG and LGG. This approach was further extended by [117] with the two-phase process along with a weighted loss function-initially, the network trains using equiprobable patches that follow actual patch training without the class imbalance problem. In [53], authors had designed 2D InputCascadedCNN, which took the output of TwoPathCNN to train other 2DCNN with the input images. After the successful implementation of FCN by the authors in [85], authors in [70] proposed two pathway architecture, where both pathways included residual connections and trained on the different input patch sizes. As the network was fully convolutional, multiple voxels of the input patch label at a time.

The [151], uses the 2D FCNN approach along with CRF. The FCNN trains on patches and CRF on slices. In [26], cascaded encoder-decoder like FCNNs along with residual connections. The first FCNN segments the whole tumor, followed by the internal tumor regions by the second FCNN. Authors in [90] had proposed encoder-decoder FCNN based architecture to segment various tumor sub-regions. In [21], authors had proposed three different FCNN architecture and showed that the architectures with multi-resolution features performed better compared to single-resolution architecture. Authors in [86] implemented Dilated Residual Network for patch based training where equiprobable patches were supplied to the network for training.

Authors in [41] adopted U-net architecture for brain tumor segmentation. Authors in [44] modified the Unet, which took 3D input, and the depth of the network reduces to three. Authors in [63], had optimized the training of the network proposed in their previous work [62]. In [27], authors had proposed novel encoder- 


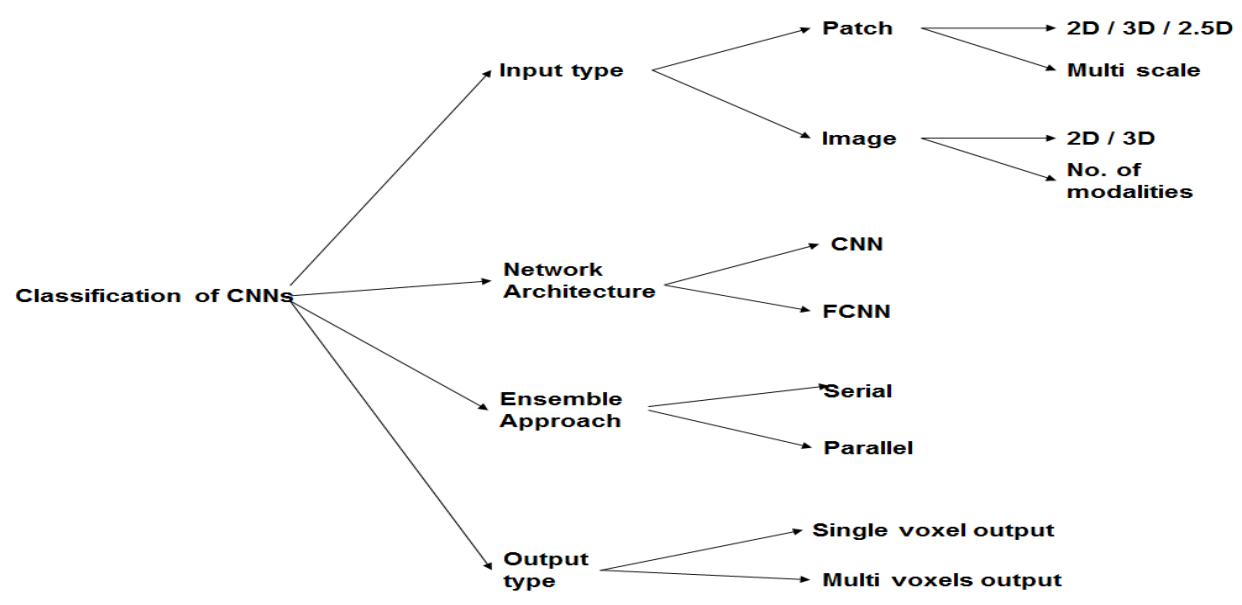

Fig. 9: Design aspects of CNN architectures.

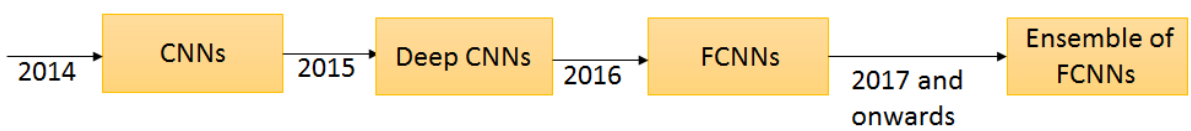

Fig. 10: Variational growth of CNN for tumor segmentation.

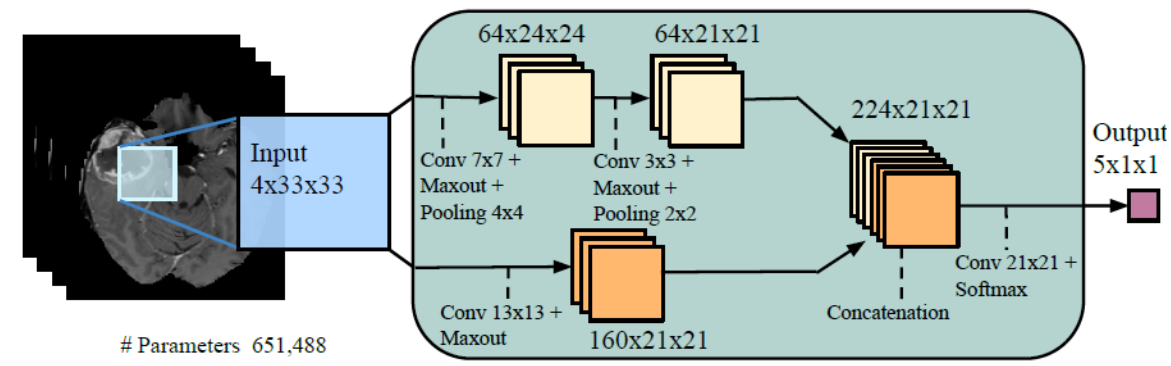

Fig. 11: TwopathwayCascadedCNN Architecture[54].

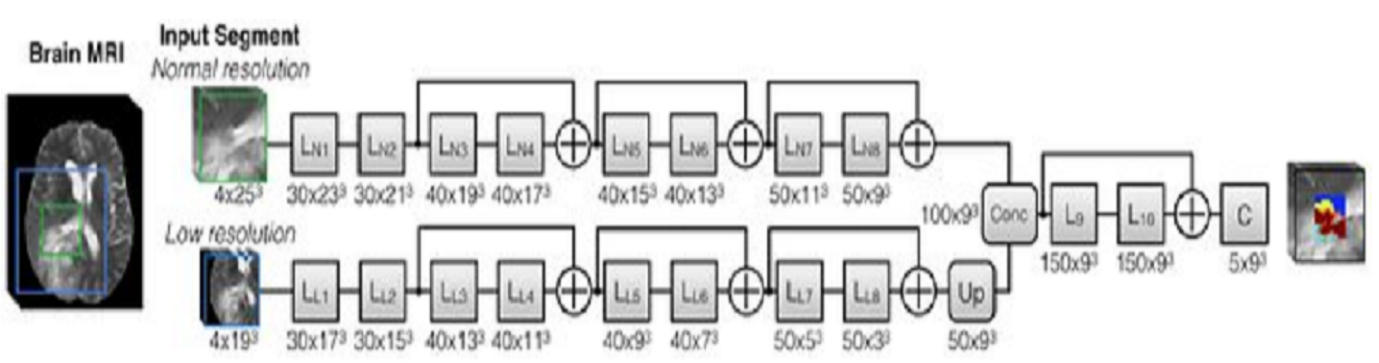

Fig. 12: Deep Medic Architecture [70]. 


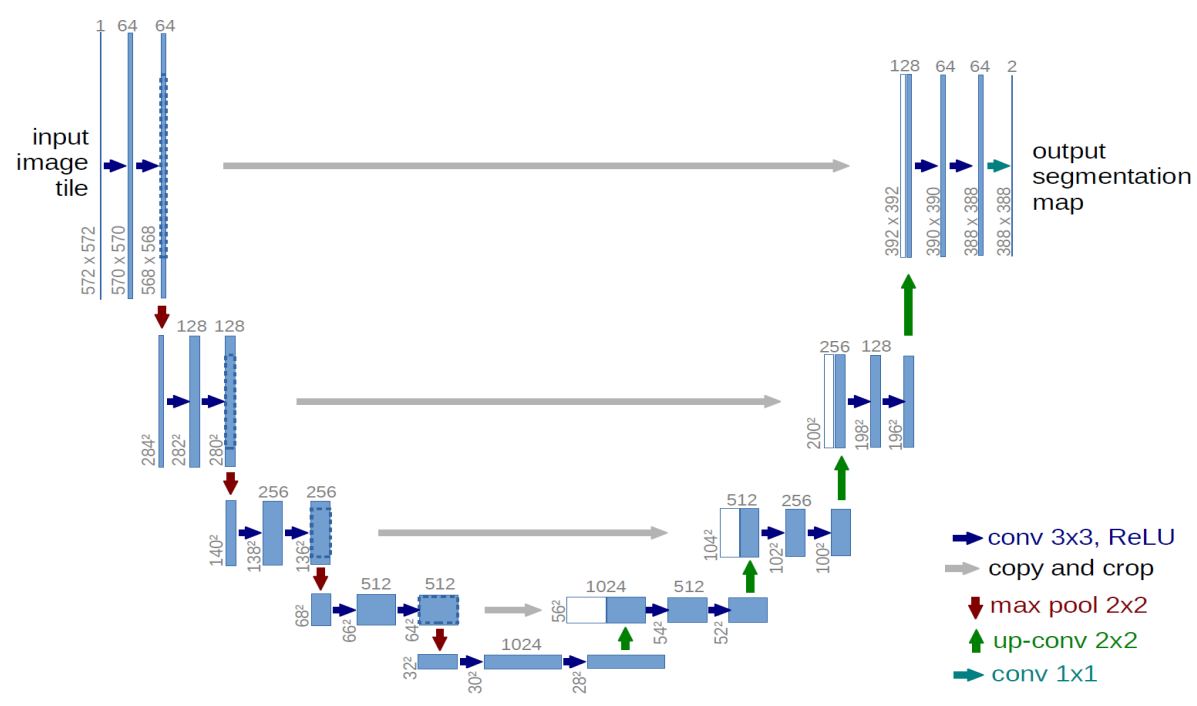

Fig. 13: U-net architecture[121].

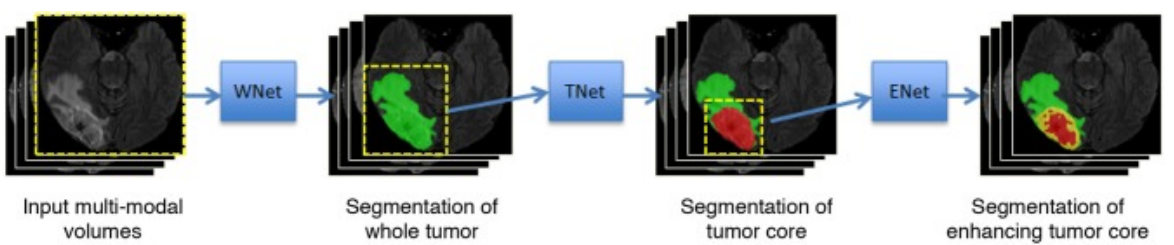

Fig. 14: Anisotropic Cascaded Architecture[142].

decoder architecture that had worked well on multiple biomedical image segmentation problems. Various other CNN based approaches where single CNN was used for segmentation task were [107], [64], [124], [111], [23], [74], [22], [67], [58], [89], [102], [94].

The ensemble of CNNs performs better compared to single CNN, as in [71], where authors had implemented an ensemble of seven networks using DeepMedic, FCN, and U-net along with variations of those three networks. They also tried different three approaches for pre-processing on all these networks. The output of individual networks with all the pre-processing generates the final label. Authors in [91] had extended their work proposed in [90] where the dense module and dilated module were introduced in the encoder decoder cascaded architecture of two networks. The pooling layers were replaced with dilated convolution layers.

The authors in [101] had implemented the ensemble of ten encoder-decoder based architectures, which included the auto-encoder stream to reconstruct the original image for additional information and regularization purpose. Authors in [92] extended their approach proposed in [69]. They used a combination of U-net and Densenet with U-net like architecture containing dense blocks of dilated convolution. The network of [151] was extended in [152] to create an ensemble of three networks which were trained on three image views. Two network cascaded path was used in [33], where Coarse Segmentation Network segmented WT and Fine Segmentation Network segmented the sub-regions of the network. Both the networks used 3D U-net with four level deep architecture.

Authors in [142] used three networks (WNet, TNet, and ENet) to prepare the cascaded path. The multiscale prediction averages for the private network. These networks train on three-views of images (axial, coronal, sagittal), and results from averages to generate the final segmentation output. In [153], the cascaded network was proposed, which initially segmented the whole tumor followed by tumor core, and enhancing tumor segmentation refinement. In [79] authors had proposed cascaded UNet with three networks. The network processed downsampled input and generated output was passed to the next network in the cascaded sequence. Authors in [146] proposed multi-scale mask 3D U-nets with atrous spatial pyramid pooling layers, where WT segmentation generated by first networks was passed to the second for TC generation, which in sequence passed 
to the final network to generate ET output. Other ensemble based CNN approaches were explained in [87], [73], [60], [143], [7], [31], [25], [147].

The comparison of the methods summarized in Table 8 is based on the pre-processing techniques, DNN architecture, activation function, loss function, postprocessing, and DSC achieved. The pre-precessing techniques considered for the comparison are:

1. Intensity Clipping: $1 \%$ of highest and lowest frequencies are clipped.

2. Bias Field Correction.

3. Z-score normalization: $Z=(x-\mu) / \sigma$.

4. Histogram matching: Histogram of all the images is match with the reference histogram.

5. Image normalization: Min-max normalization.

6. Intensity standardization with Nyul approach [104].

7. Image denoising: applies noise filtering for e.g. Gaussian noise filtering.

8. Intensity rescaling : rescaling the intensity range between some specific limits.

The post-processing techniques used for segmentation result improvement are:

1. Connected Component Analysis: Analyse the connected components and removes the component with the volume below some threshold.

2. Conditional Random Field.

3. Morphological Operators to remove false positives and fill the holes

4. relabelling the output label: Enhancing tumor labels below some threshold are relabelled as necrosis.

Fig. 15 shows the pictorial representation of the DSC of various CNN methods for whole tumor segmentation. The average DSC of CNN is 0.86 , deep CNN and FCN is 0.87 , and for ensemble approach is 0.89 . The ensemble of the CNNs/FCNNs learns the robust features from the input.

\subsection{Hardware and Software for DNN}

Hardware: DNN methods have gained its popularity after availability of the Graphical Processing Units (GPU). Nowadays efficient parallel processing for manipulation of large amount of data is applicable with help of General Purpose GPU(GPGPU). The computing libraries like CUDA and OpenGL allows the efficient implementation of the processing code on GPU. The performance of the GPU highly depends on GPU computing cores (CUDA cores), Tensor processing cores, Thermal Design Power (TDP), and on board GPU memory. Various types of GPUs used for the implementation of the CNN methods for segmentation task are as shown in Table 7. As the computing capacity of the GPU increases, it allows more complex networks with higher number of network parameters to be trained with less time.

Table 7: GPU specifications[103].

\begin{tabular}{|c|c|c|c|c|c|}
\hline Year & $\begin{array}{l}\text { GPU } \\
\text { Type }\end{array}$ & $\begin{array}{l}\text { CUDA } \\
\text { Cores }\end{array}$ & $\begin{array}{l}\text { Tensor } \\
\text { Cores }\end{array}$ & $\begin{array}{l}\text { TDP } \\
\text { (Watts) }\end{array}$ & $\begin{array}{l}\text { RAM } \\
\text { (GB) }\end{array}$ \\
\hline 2016 & Tesla K80 & 2496 & $\mathrm{~N} / \mathrm{A}$ & 300 & 12 \\
\hline 2016 & $\begin{array}{l}\text { GeForce } \\
\text { GTX } 980 \\
\text { Ti }\end{array}$ & 2816 & $\mathrm{~N} / \mathrm{A}$ & 165 & 6 \\
\hline $\begin{array}{l}2016 \\
2017\end{array}$ & $\begin{array}{l}\text { - } \quad \text { GeForce } \\
\text { GTX } 1080 \\
\text { Ti }\end{array}$ & 3584 & $\mathrm{~N} / \mathrm{A}$ & 250 & 11 \\
\hline 2017 & $\begin{array}{l}\text { GTX } \\
\text { Titan X }\end{array}$ & 3072 & $\mathrm{~N} / \mathrm{A}$ & 240 & 12 \\
\hline 2018 & $\begin{array}{l}\text { Quadro } \\
\text { P4000 }\end{array}$ & 1792 & $\mathrm{~N} / \mathrm{A}$ & 105 & 8 \\
\hline 2018 & $\begin{array}{l}\text { Quadro } \\
\text { P5000 }\end{array}$ & 2560 & $\mathrm{~N} / \mathrm{A}$ & 180 & 16 \\
\hline 2018 & Titan Xp & 3840 & $\mathrm{~N} / \mathrm{A}$ & 250 & 12 \\
\hline 2018 & Tesla P100 & 3584 & $\mathrm{~N} / \mathrm{A}$ & 250 & 16 \\
\hline 2018 & $\begin{array}{l}\text { Tesla } \\
\text { V100 }\end{array}$ & 5120 & 640 & 300 & $16 / 32$ \\
\hline 2018 & DGX-1 & $\begin{array}{l}40960 \\
(8 \times V 100)\end{array}$ & 5120 & 3500 & $\begin{array}{l}128(16 \\
\text { per } \\
\text { GPU })\end{array}$ \\
\hline
\end{tabular}

Software: The open software library packages provide implementation of various neural network operations like convolution. Most popular python library packages used for DNN method implementations are Caffe[68], Tensorflow[1], Theano[16], and PyTorch[106]. Some of the third-party packages which work on the top of these networks are Keras[30], Lasagne[39], and TensorLayer[40]. 


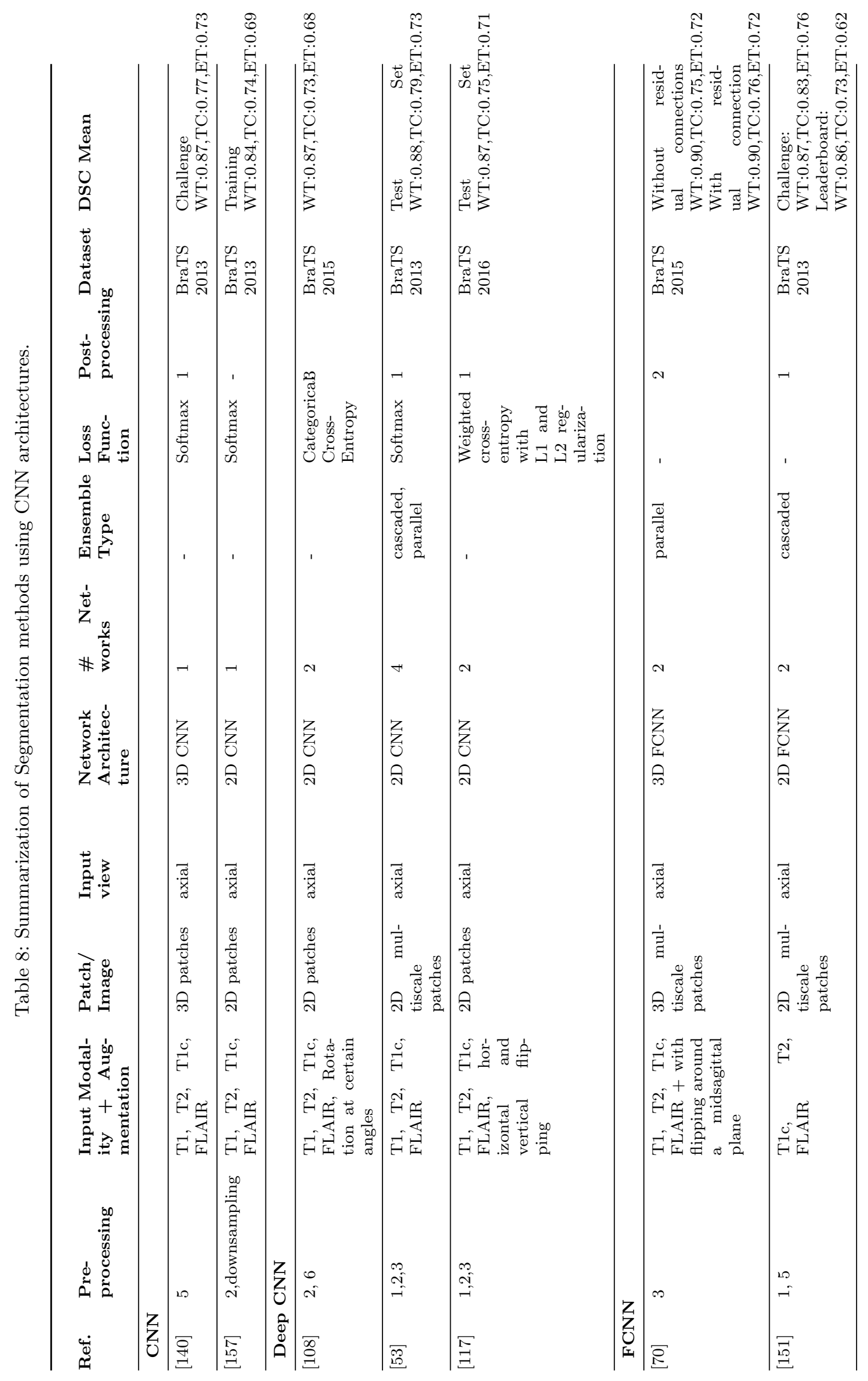




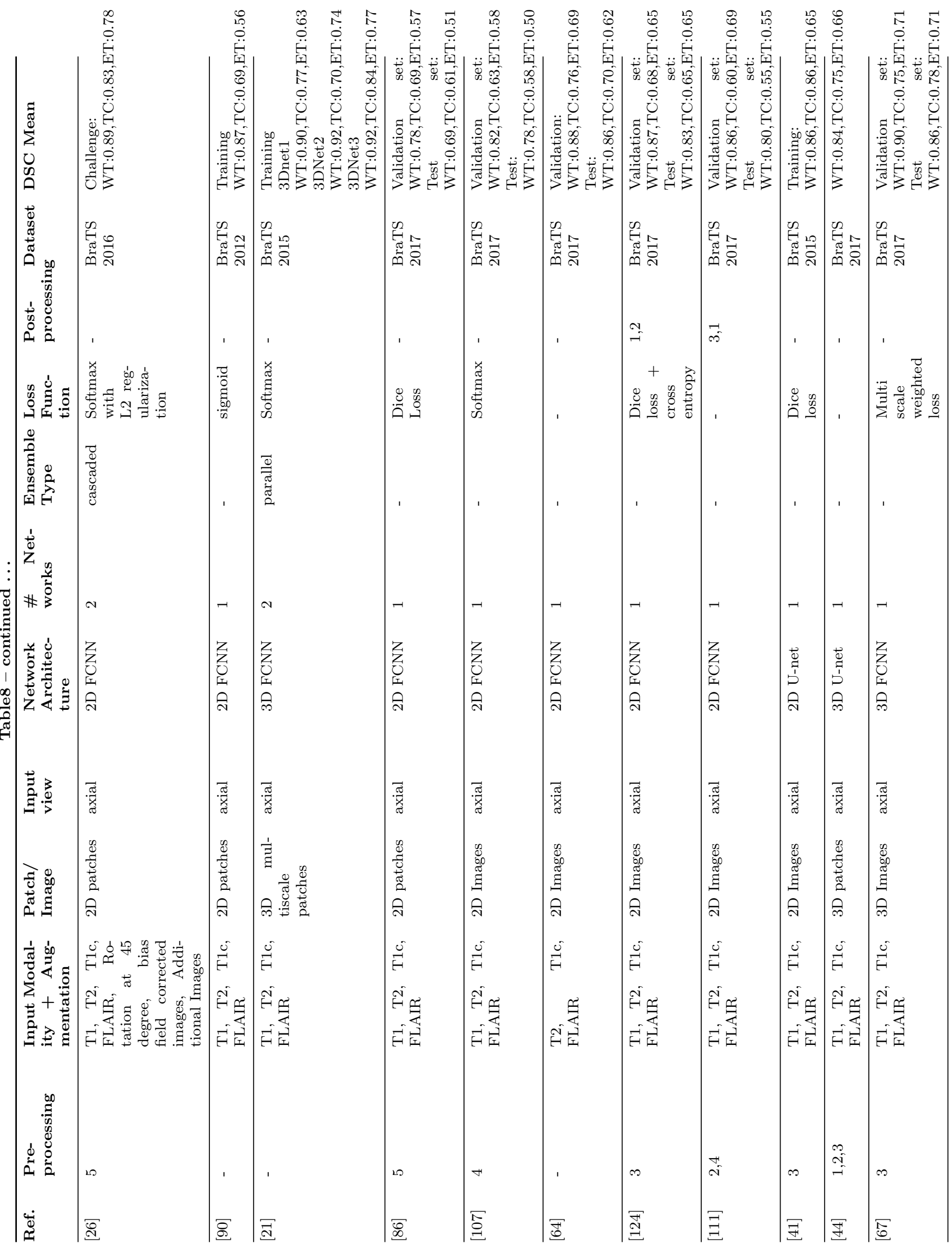




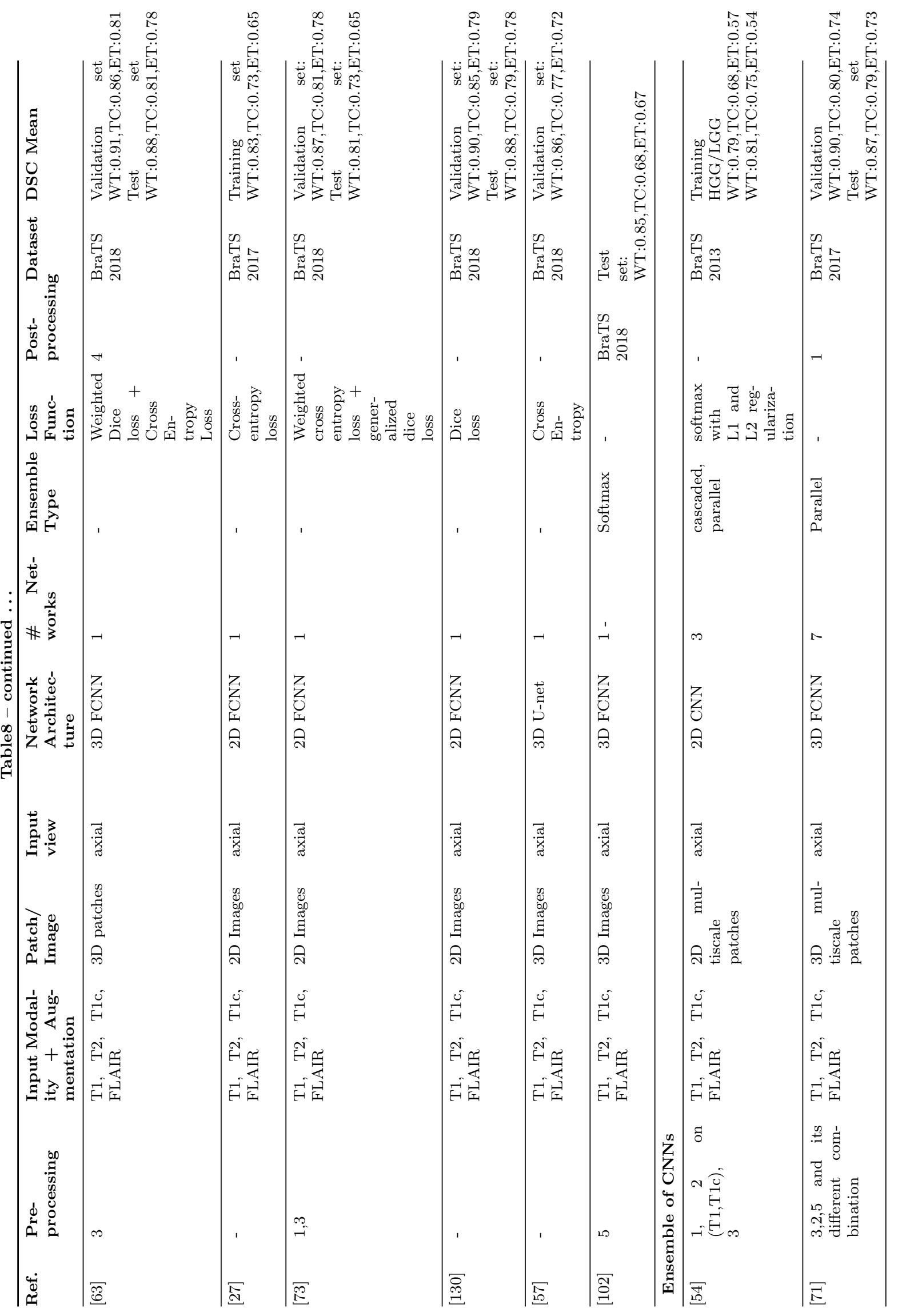




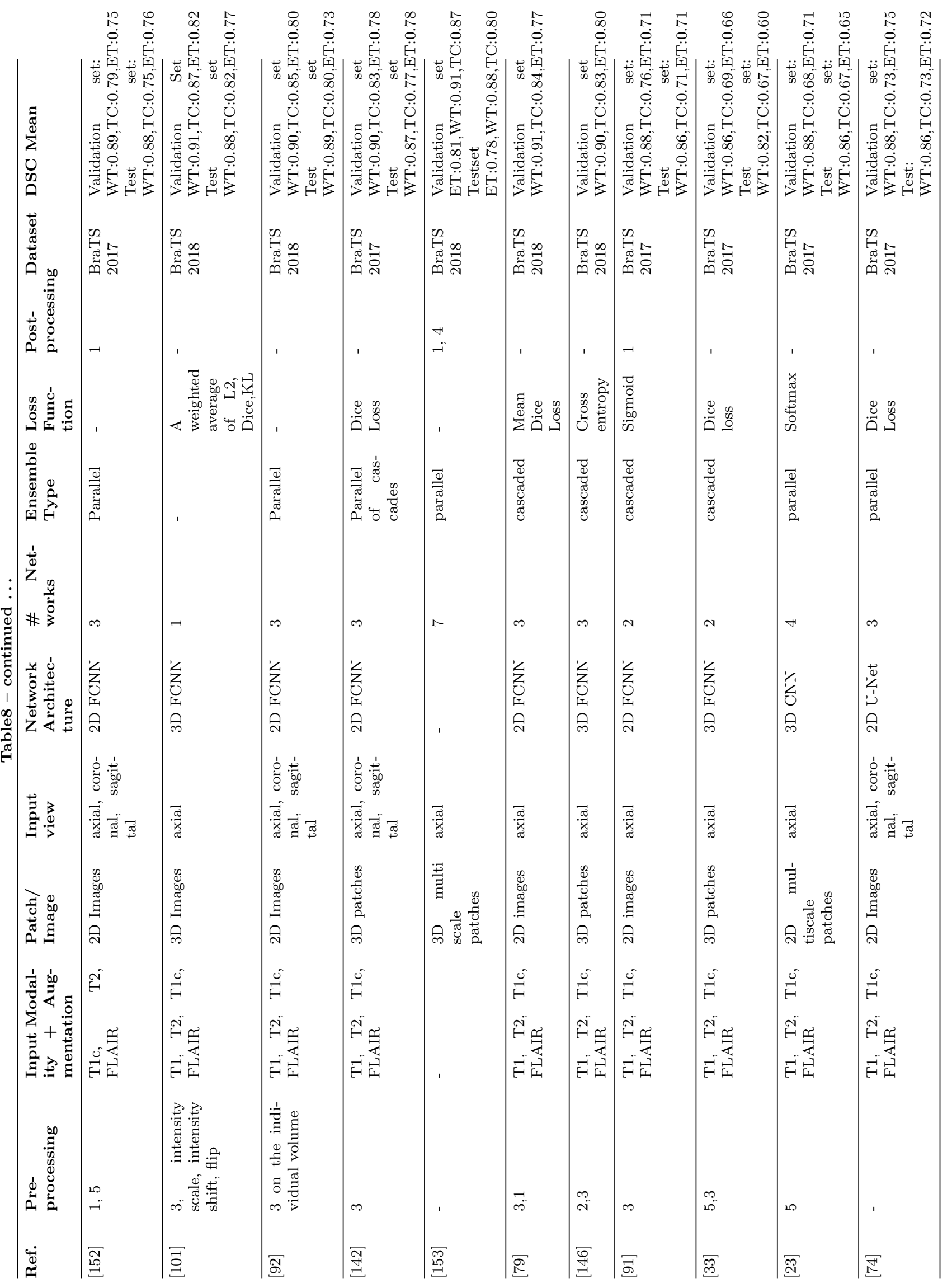




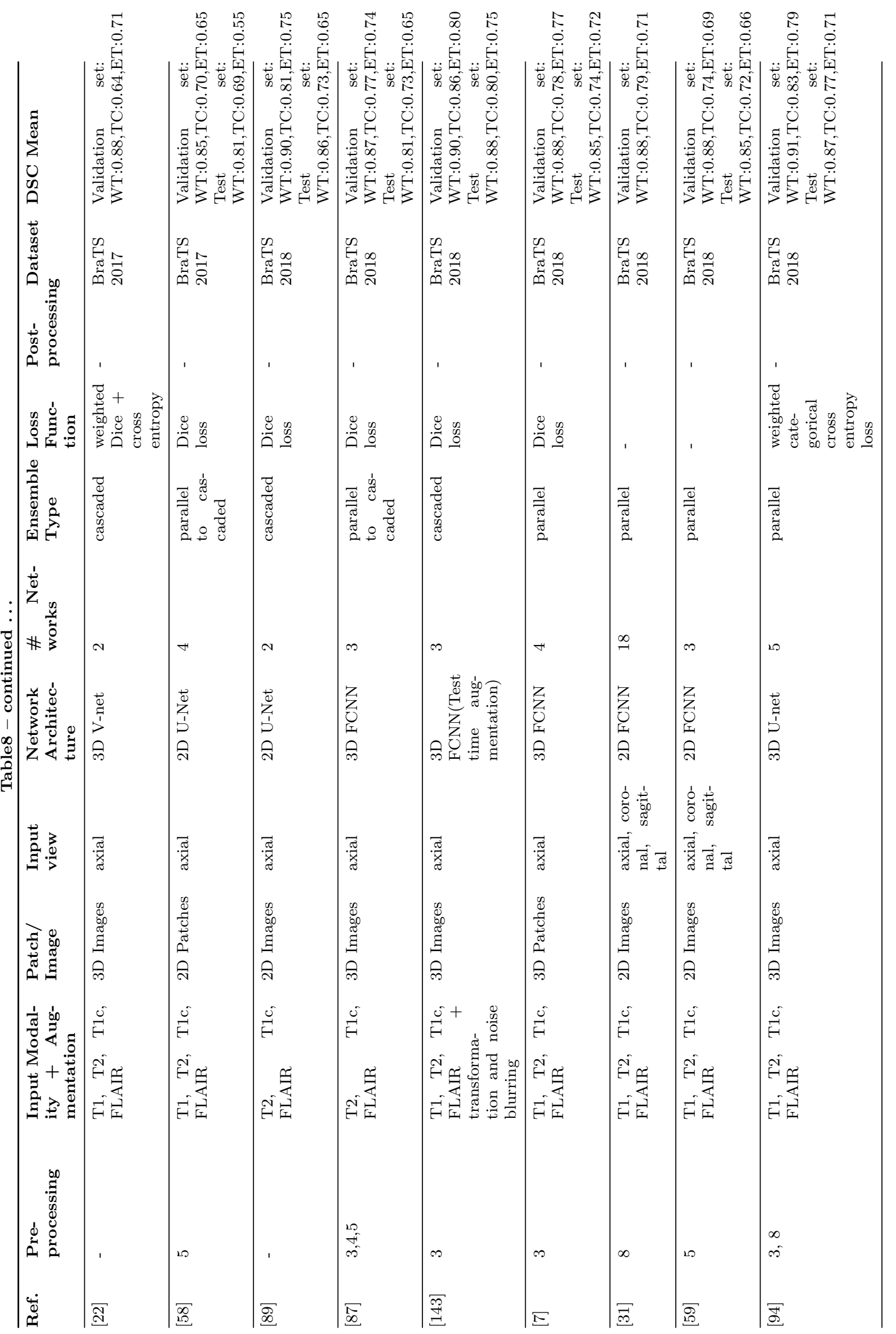




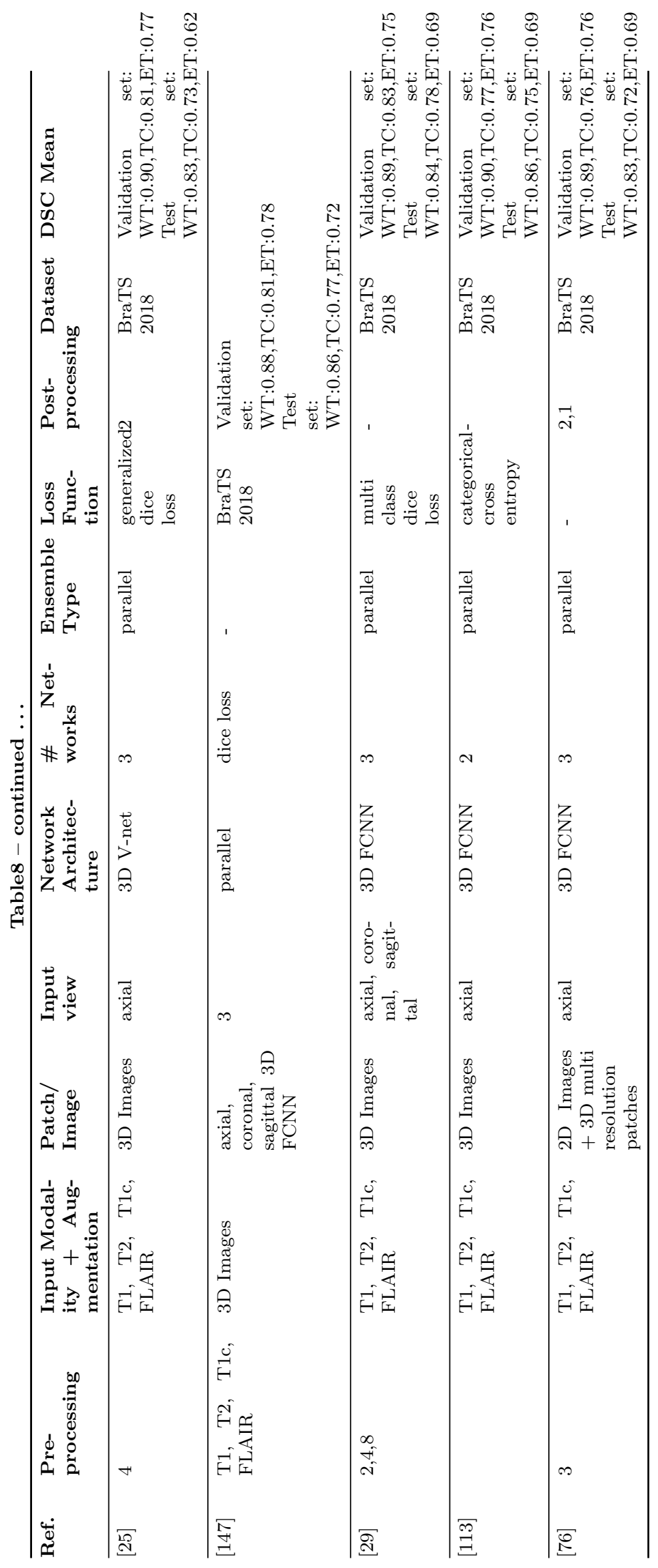




\subsection{Proposed Architecture for Tumor Segmentation}

The authors of the article have adopted 2D U-net architecture with three layers[3]. Each encoder layer is replaced with a dense module as shown in Fig 16. In the first phase, the network is trained for whole tumor for 50 epochs with dice loss function. Further the network parameters are initialized with whole tumor weights to train the network for necrosis, enhancing and edema subregions. The inductive transfer learning approach [105] for parameter initialization has reduced the false positives and boosted the network training convergence. In the second phase, these subregion weights are used as initial parameters to train the networks again for the same subregions using focal loss function. The network training continues for 50 epochs for each subregions. From the BraTS 2019 training dataset, 85\% of the images are given for training and $15 \%$ are kept for validation. The input to the network is $2 \mathrm{D}$ slices of $240 \times 240$ size from all the four modalities. The blank slices of the dataset are removed as it does not contain any meaningful information. The following are the updates applied to the network:

- The bounding box is applied around the brain to reduce the input which contains zero intensity (blank) voxels.

- The convolution layer is added in the dense module.

The results for the network are in Table 9, which includes minor variations in the network, different input image sizes, different loss functions, and the number of images in training. The network is trained on $85 \%$ of the total image and validated on the remaining $15 \%$ images of the BraTS 2019 dataset. The online tool provided by the organizer generates the evaluation metric for the training set as well as a separate validation set in addition to the training set.

The DSC comparison of training and validation sets for these variations are as shown in Fig. 17. The segmentation results show that the method overfits the training data and does not generate good results for the unknown validation set. The segmentation results of the sample images from the training set for correct as well as incorrect segmentation are shown in Fig. 18 and Fig. 19. The network does not distinguish between the subregions where the tumor appearance is homogeneous for all its subregions and intensity is same as the normal brain tissues.
5.6 End to End methods for tumor segmentation and OS prediction

Since 2017, the second task of survival prediction was introduced in the challenge. Some of the methods had participated in end-to-end solutions in the challenge, i.e., segmentation followed by survival prediction.

In [125], an ensemble of RF and CNN segments the tumor and random forest regressor(RFR) was used to predict the overall survival days using 240 features out of 1366 different features (Kaplan-Meier was used to find the relevant and useful features). Authors in [69] had modified U-net with Full Resolution Residual Network(FRRN) and Residual Unit(RU) units along with weight scaling dropout. The survival prediction ANN worked with linear activation function on four selected features.

The variant of U-net was used in [62], which took $3 \mathrm{D}$ input and included context module and localization module in each level of the architecture. The segmentation result was generated based on the elementwise summation of the output from the decoder layers. Survival prediction was the average of RFR and multilayer perceptron(MLP). RFR trains on 517 features extracted from three tumor sub-regions using the radiomics package[141]. The output of RFR and MLP averages 15 MLPs design with three hidden layers, each with 64 neurons.

Authors in [9] implemented 3D U-net with three stages of encoder-decoder architecture. Regression model based radiomics features selection trains MLP for OS prediction. Whereas in [144], two 3D U-nets uses fourstage encoder-decoder architecture, the first network segmented the whole tumor, and the second one segmented the tumor sub-region. In addition to four conventional modalities, they used the additional image as an input, which is the T1c-T1 subtracted image. This image provides additional information for the tumor core region. They used only the age feature was used to predict OS using linear regressor. The approach presented in [83] used FCNN named FCRN-101, which derives from pre-trained SegNet and U-net architecture. Three path network combines the result of three views, i.e., axial, coronal, and sagittal. The OS prediction used SPNet, the fully-connected CNN, which took four modalities and the network segmentation result as input to predict the probability of OS prediction.

The [45], uses an ensemble of six 3D U-net type networks with variation in the input size, number of encoding/decoding blocks, and feature maps at every layer. The OS prediction uses linear regression with ground truth image volume, surface area, age, and resection status. Features were input to the network after z-score 


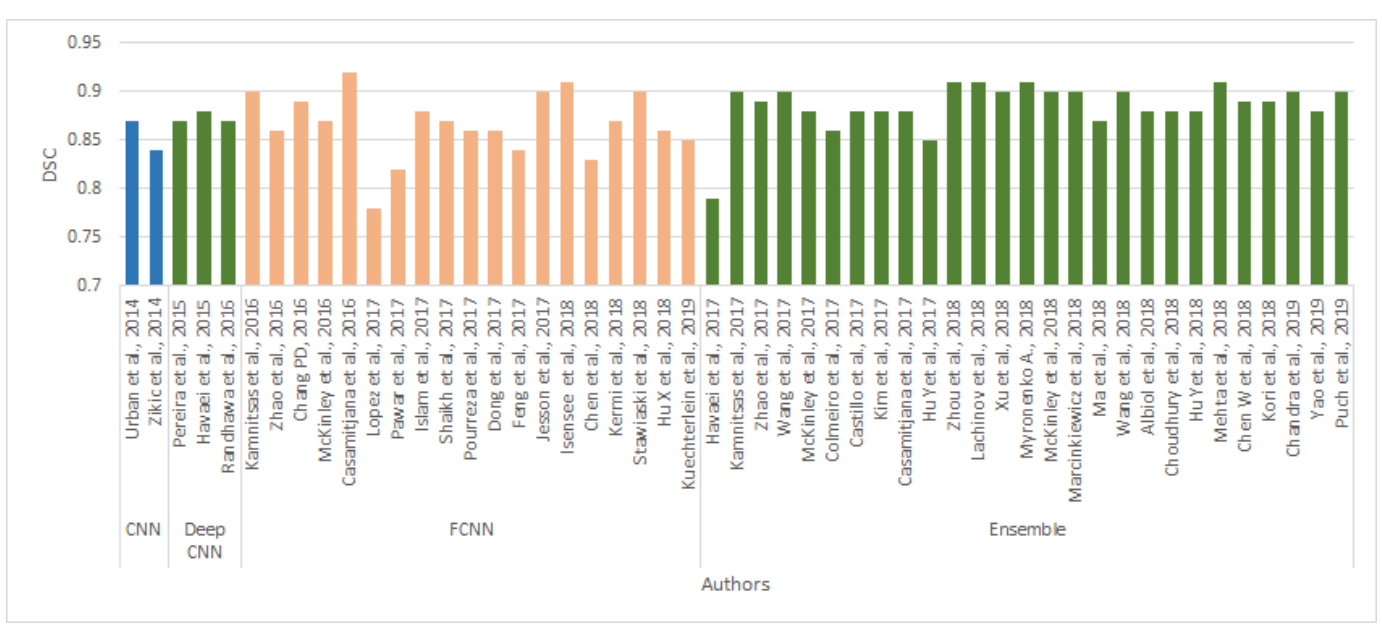

Fig. 15: DSC comparison for whole tumor segmentation of CNN methods.
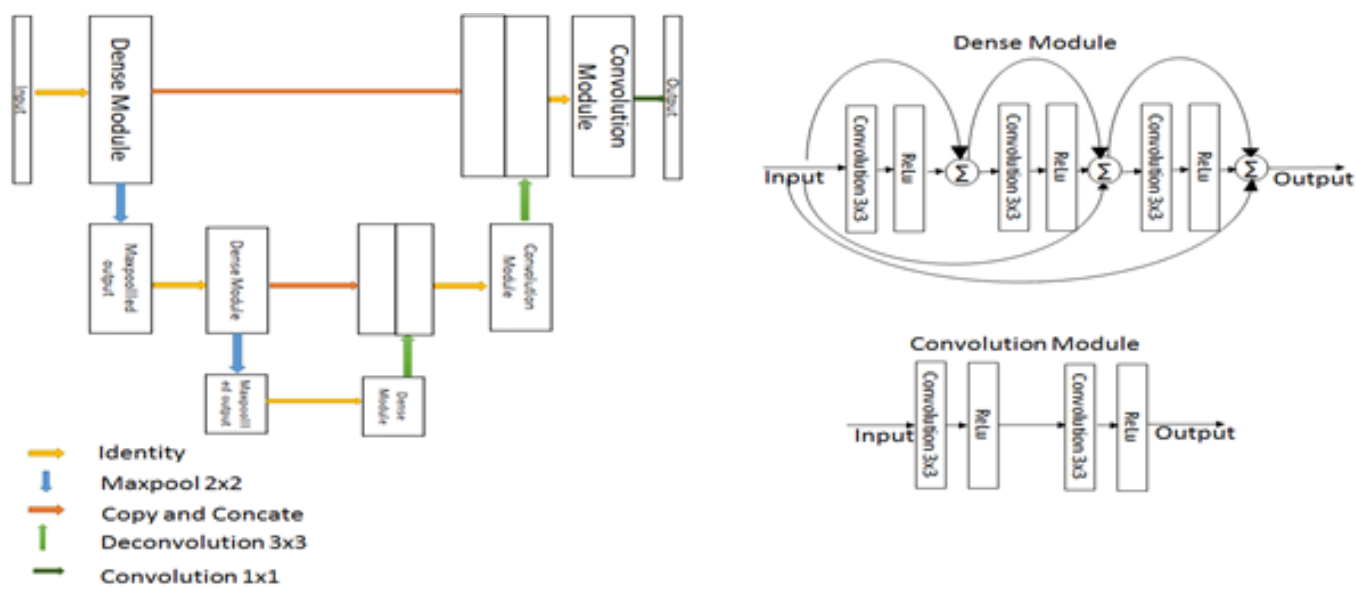

Fig. 16: Three stage U-net architecture [3].

normalization. Authors in [114] had implemented FCN and generated results for three axes and used majority voting to generate the final segmentation results. For OS prediction, ten features (focusing on necrosis and active tumor) from the segmentation results were generated, and mean PCA and standard deviation PCA to train RF on the GTR images.

In [133] an ensemble of three networks (U-net[121], DFKZNet[62], and CA-CNN[142]) was used, and majority voting applied for final segmentation. The OS prediction used RF with 14 radiomics features selected from various modality images, Laplacian of Gaussian Images and wavelet decomposed images. Authors in [6] had implemented 2D U-net architecture with three stages for tumor segmentation and age, volumetric, and shape features of the whole tumor were used to predict OS.
All the approaches did not use the location-based information of the tumor and its sub-regions. In contrast, [72], uses twenty-one brain parcellation regions as an input along with the four MR modalities. It emphasizes the number of tumor regions in those specific parcellation areas. Those twenty-five input channels were given as input the ensemble of 3D U-net as well as the ensemble of DeepMedic architectures with different kernel and input patch sizes. Tractrographic features from network segmented regions trains SVM classifiers with the linear kernel to predict OS. Authors in [3] implements 2D U-net of three stages with dense blocks at every encoder level, and the feature set of [6] of necrosis tumor sub-region for OS prediction.

The authors in [128] combines the features from VGG16 based FCN and texton maps to generate the features and supply them to RF classifier to generate the segmentation result. RF is also used for OS pre- 
Table 9: Comparison of model variations of [3].

\begin{tabular}{|c|c|c|c|c|c|c|c|}
\hline Model & $\begin{array}{l}\text { Architectural } \\
\text { change }\end{array}$ & $\begin{array}{l}\text { Input } \\
\text { Modalities }\end{array}$ & $\begin{array}{l}\# \text { Feature } \\
\text { maps }\end{array}$ & $\begin{array}{l}\text { Input im- } \\
\text { age size }\end{array}$ & $\begin{array}{l}\text { Loss func- } \\
\text { tion }\end{array}$ & $\begin{array}{l}\text { DSC } \\
\text { Training } \\
\text { Set }\end{array}$ & $\begin{array}{l}\text { DSC Vali- } \\
\text { dation Set }\end{array}$ \\
\hline 1 & Original & $\begin{array}{l}\text { T1, T1c, T2, } \\
\text { FLAIR }\end{array}$ & $\begin{array}{l}32,64,128 \\
64,32\end{array}$ & $240 \times 240$ & Dice Loss & $\begin{array}{l}\text { WT:0.90 } \\
\text { TC:0.86 } \\
\text { ET: } 0.75\end{array}$ & $\begin{array}{l}\text { WT:0.72 } \\
\text { TC:0.63 } \\
\text { ET:0.55 }\end{array}$ \\
\hline 2 & Original & $\begin{array}{l}\text { T1, T1c, T2, } \\
\text { FLAIR }\end{array}$ & $\begin{array}{l}32,64,128 \\
64,32\end{array}$ & $156 \times 200$ & Dice Loss & $\begin{array}{l}\text { WT: } 0.90 \\
\text { TC:0.86 } \\
\text { ET: } 0.75\end{array}$ & $\begin{array}{l}\text { WT:0.72 } \\
\text { TC:0.63 } \\
\text { ET:0.55 }\end{array}$ \\
\hline 3 & $\begin{array}{l}\text { dense module } \\
+\quad \text { convolution } \\
\text { layer }\end{array}$ & $\begin{array}{l}\text { T1, T1c, T2, } \\
\text { FLAIR }\end{array}$ & $\begin{array}{l}32,64,128 \\
64,32\end{array}$ & $156 \times 200$ & Dice Loss & $\begin{array}{l}\text { WT:0.89 } \\
\text { TC:0.84 } \\
\text { ET:0.71 }\end{array}$ & $\begin{array}{l}\text { WT:0.71 } \\
\text { TC:0.61 } \\
\text { ET:0.56 }\end{array}$ \\
\hline 4 & $\begin{array}{l}\text { dense module } \\
+\quad \text { convolution } \\
\text { layer }\end{array}$ & $\begin{array}{l}\text { T1, T1c, T2, } \\
\text { FLAIR }\end{array}$ & $\begin{array}{l}32,64,128 \\
64,32\end{array}$ & $156 \times 200$ & Focal Loss & $\begin{array}{l}\text { WT:0.93 } \\
\text { TC:0.90 } \\
\text { ET: } 0.83\end{array}$ & $\begin{array}{l}\text { WT:0.75 } \\
\text { TC:0.65 } \\
\text { ET:0.60 }\end{array}$ \\
\hline 5 & $\begin{array}{l}\text { dense module } \\
+\quad \text { convolution } \\
\text { layer }\end{array}$ & $\begin{array}{l}\text { T1c, } \\
\text { FLAIR }\end{array}$ & $\begin{array}{l}32,64,128 \\
64,32\end{array}$ & $156 \times 200$ & Dice Loss & $\begin{array}{l}\text { WT: } 0.87 \\
\text { TC:0.81 } \\
\text { ET:0.67 }\end{array}$ & $\begin{array}{l}\text { WT: } 0.71 \\
\text { TC: } 0.58 \\
\text { ET: } 0.51\end{array}$ \\
\hline 6 & $\begin{array}{l}\text { dense module } \\
+\quad \text { convolution } \\
\text { layer }\end{array}$ & $\begin{array}{l}\text { T1c, } \\
\text { FLAIR }\end{array}$ & $\begin{array}{l}32,64,128 \\
64,32\end{array}$ & $156 \times 200$ & Focal Loss & $\begin{array}{l}\text { WT: } 0.92 \\
\text { TC:0.89 } \\
\text { ET: } 0.79\end{array}$ & $\begin{array}{l}\text { WT: } 0.76 \\
\text { TC:0.66 } \\
\text { ET: } 0.60\end{array}$ \\
\hline
\end{tabular}

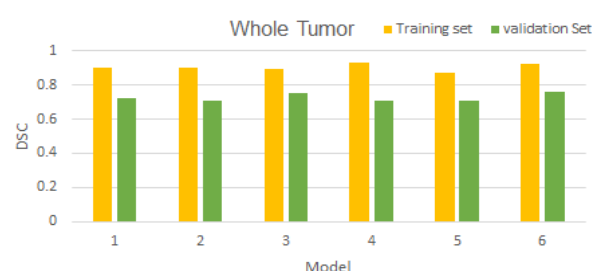

(a)

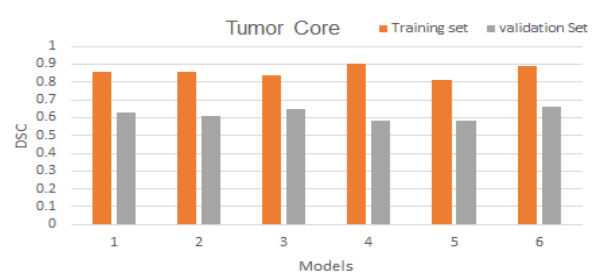

(b)

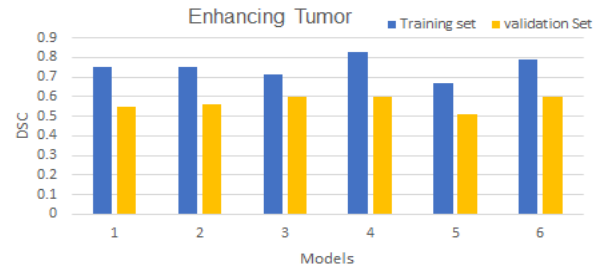

(c)

Fig. 17: DSC comparison for training and validation set a) whole tumor b)tumor core c) ehhancing tumor. diction using volumetric as well as age feature of the patient.

Authors in [154], [60], [65], [37] attempts the endto-end segmentation approach.

Table 10 compares the segmentation results of endto-end methods. The approaches for pre-processing and post-processing techniques are as specified in section 5.3 and Table 11 provides details related to the survival prediction. 


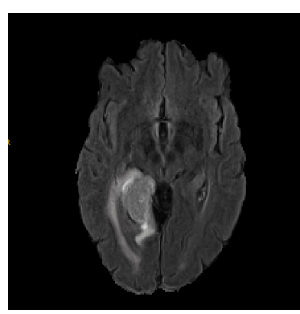

(a)

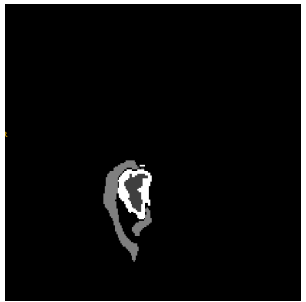

(c)

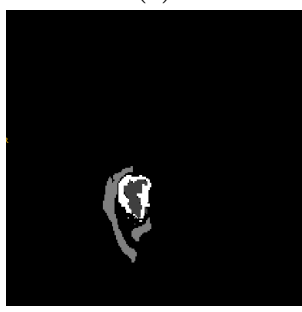

(e)

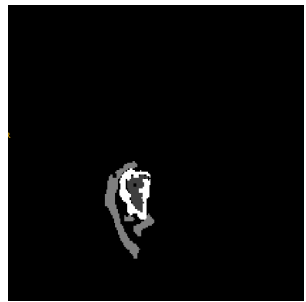

(g)

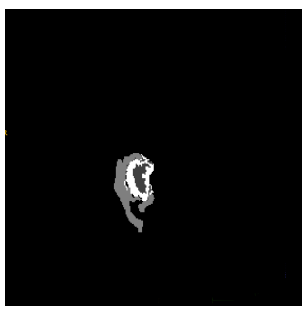

(b)

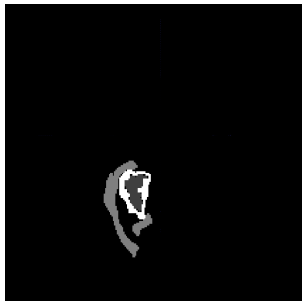

(d)

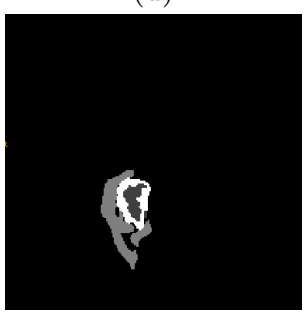

(f)

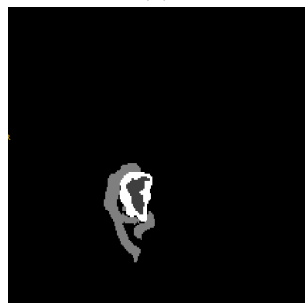

(h)

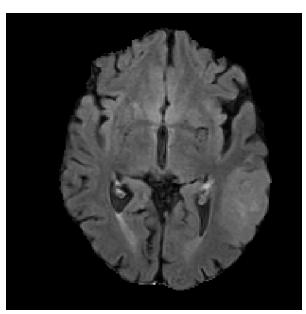

(a)

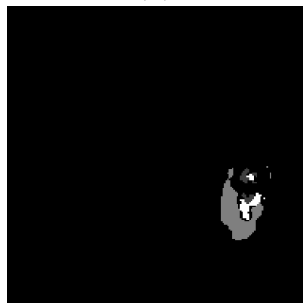

(c)

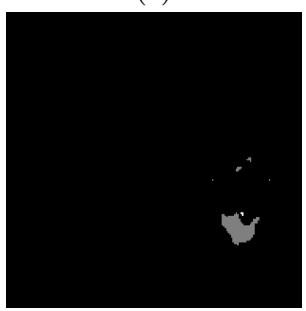

(e)

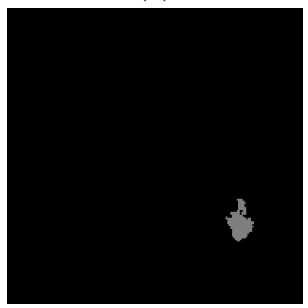

(g)

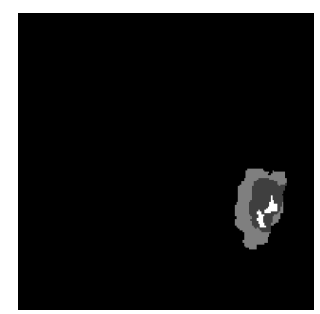

(b)

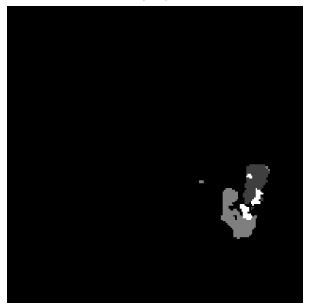

(d)

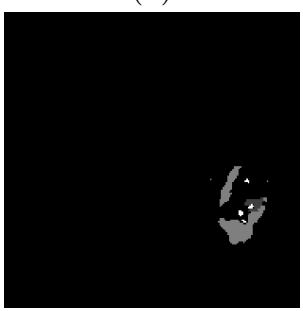

(f)

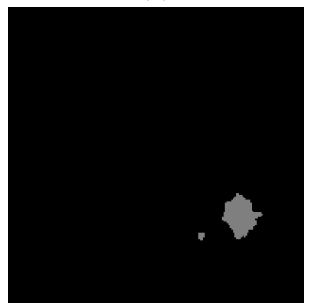

(h)

Fig. 18: Training set image, correct segmentation a) Original FLAIR image b) ground truth segmentation c) model $1 \mathrm{~d}$ ) model 2 e) model $3 \mathrm{f}$ ) model $4 \mathrm{~g}$ ) model $5 \mathrm{~h}$ ) model 6.

Fig. 19: Training set image, incorrect segmentation a) Original FLAIR image b) ground truth segmentation c) model $1 \mathrm{~d}$ ) model 2 e) model $3 \mathrm{f}$ ) model g) model 5 h) model 6 . 


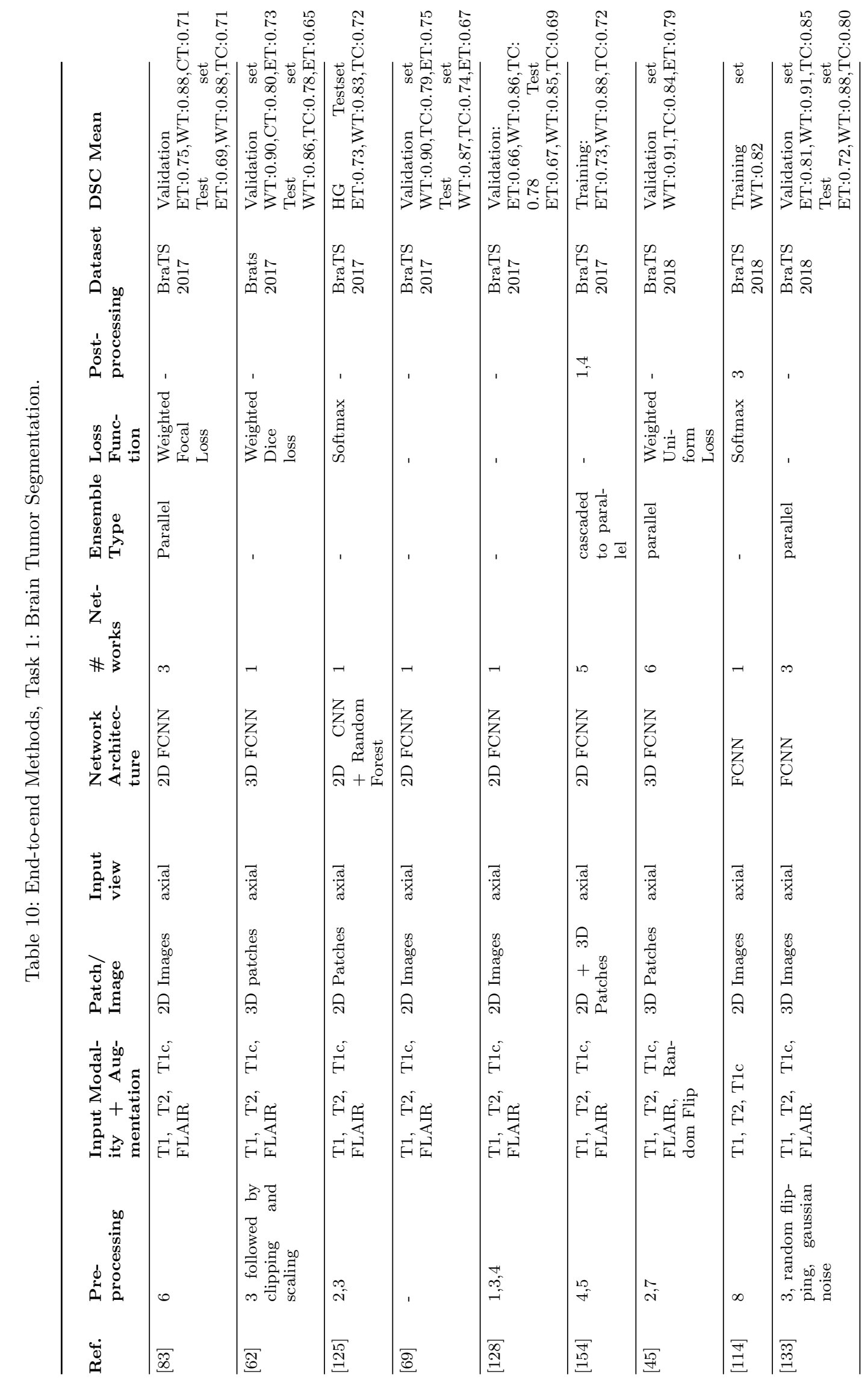




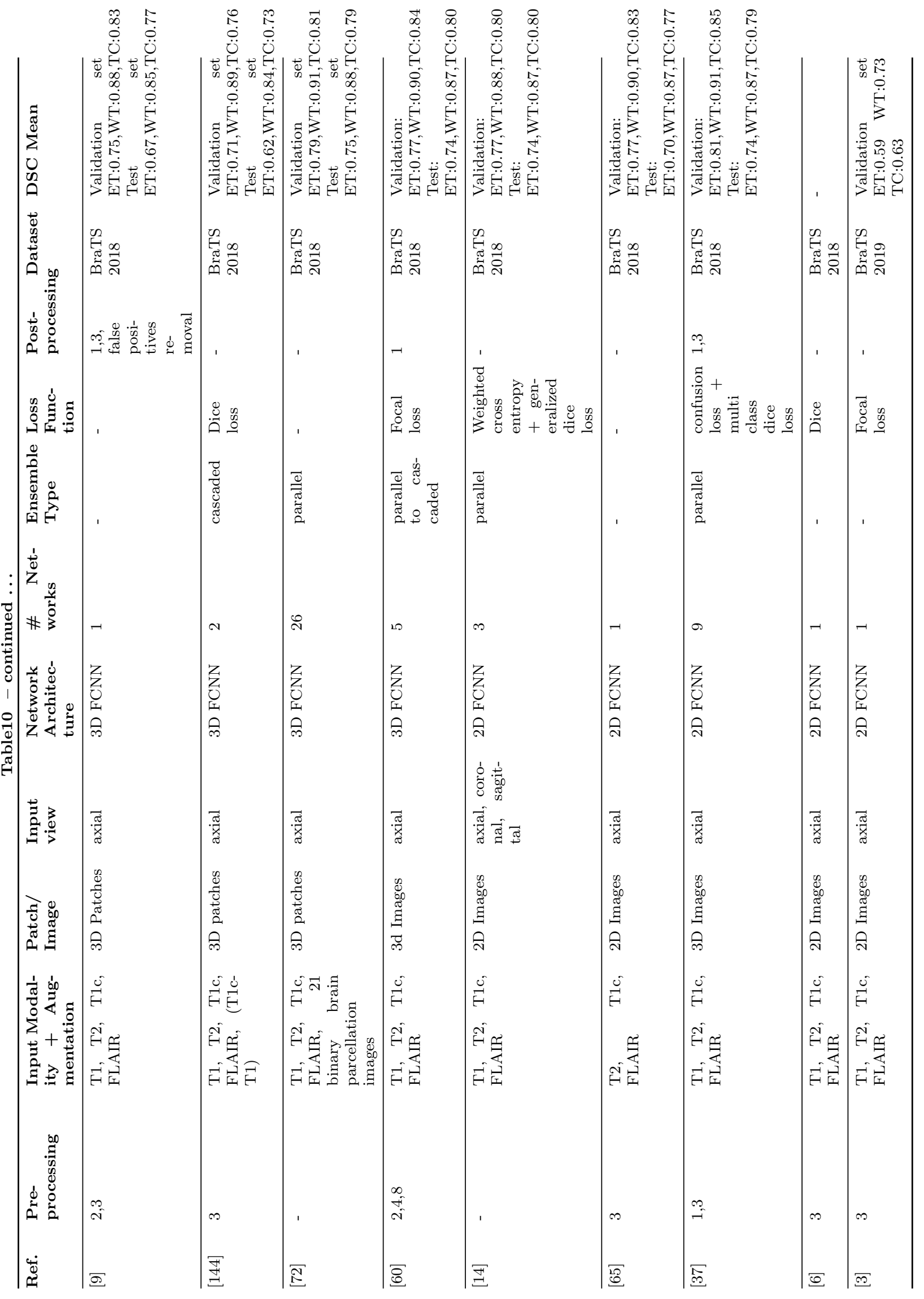


Table 11: End-to-end Methods, Task 2: OS Prediction.

\begin{tabular}{|c|c|c|c|c|}
\hline Ref. & Method & $\begin{array}{l}\# \quad \text { fea- } \\
\text { tures/type } \\
\text { of net- } \\
\text { work }\end{array}$ & Dataset & $\begin{array}{l}\text { Accuracy } \\
(\%)\end{array}$ \\
\hline [83] & $2 \mathrm{D} \mathrm{CNN}$ & - & $\begin{array}{l}\text { BraTS } \\
2017\end{array}$ & $\begin{array}{l}\text { Valid:55 } \\
\text { Test: } 45\end{array}$ \\
\hline$[62]$ & $\begin{array}{l}\text { RFR } \\
\text { MLP }\end{array}$ & 66 & $\begin{array}{l}\text { BraTS } \\
2017\end{array}$ & 52.6 \\
\hline$[125]$ & RFR & 240 & $\begin{array}{l}\text { BraTS } \\
2017\end{array}$ & $\begin{array}{l}\text { Valid:66.7 } \\
\text { Test: } 57.9\end{array}$ \\
\hline$[69]$ & ANN & 4 & $\begin{array}{l}\text { BraTS } \\
2017\end{array}$ & $\begin{array}{l}\text { Valid: } 42.4 \\
\text { Test: } 56.8\end{array}$ \\
\hline$[45]$ & $\begin{array}{l}\text { Linear Regres- } \\
\text { sion Model }\end{array}$ & 9 & $\begin{array}{l}\text { BraTS } \\
2018\end{array}$ & Valid:32.1 \\
\hline$[114]$ & $\mathrm{PCA}+\mathrm{RF}$ & 10 & $\begin{array}{l}\text { BraTS } \\
2018\end{array}$ & Test:61 \\
\hline [133] & $\mathrm{RF}$ & 14 & $\begin{array}{l}\text { BraTS } \\
2018\end{array}$ & $\begin{array}{l}\text { Valid:46.4 } \\
\text { Test: } 61 \\
\text { Second } \\
\text { Rank }\end{array}$ \\
\hline$[9]$ & MLP & 468 & $\begin{array}{l}\text { BraTS } \\
2018\end{array}$ & $\begin{array}{l}\text { Valid:57.1 } \\
\text { Test:55.8 }\end{array}$ \\
\hline [144] & $\begin{array}{l}\text { Linear Regres- } \\
\text { sor }\end{array}$ & 1 & $\begin{array}{l}\text { BraTS } \\
2018\end{array}$ & $\begin{array}{l}\text { Valid:50 } \\
\text { Test:55.8 }\end{array}$ \\
\hline$[72]$ & $\begin{array}{l}\text { SVM with lin- } \\
\text { ear kernel }\end{array}$ & - & $\begin{array}{l}\text { BraTS } \\
2018\end{array}$ & $\begin{array}{l}\text { Valid:35.7 } \\
\text { Test: } 41.6\end{array}$ \\
\hline$[6]$ & $\mathrm{RF}$ & 13 & $\begin{array}{l}\text { BraTS } \\
2018\end{array}$ & 59 \\
\hline [3] & $\mathrm{RF}$ & 13 & $\begin{array}{l}\text { BraTS } \\
2019\end{array}$ & $\begin{array}{l}\text { Valid:58.6 } \\
\text { Test:57.9 }\end{array}$ \\
\hline$[128]$ & $\mathrm{RF}$ & 4 & $\begin{array}{l}\text { BraTS } \\
2017\end{array}$ & $\begin{array}{l}\text { Valid:48.5 } \\
\text { Test: } 41.1\end{array}$ \\
\hline$[154]$ & $\mathrm{CNN}+\mathrm{XBoost}$ & $\begin{array}{l}183+ \\
\text { CNN } \\
\text { features }\end{array}$ & $\begin{array}{l}\text { BraTS } \\
2017\end{array}$ & Training: 6 \\
\hline$[60]$ & $\begin{array}{l}\text { ensemble of } \\
\text { Xboost, SVM, } \\
\text { MLP, DT, } \\
\text { RF, LDA }\end{array}$ & 900 & $\begin{array}{l}\text { BraTS } \\
2018\end{array}$ & Test:51.9 \\
\hline$[14]$ & MLP & 83 & $\begin{array}{l}\text { BraTS } \\
2018\end{array}$ & Valid:54 \\
\hline$[65]$ & ANN & 50 & $\begin{array}{l}\text { BraTS } \\
2018\end{array}$ & $\begin{array}{l}\text { Valid:67.9 } \\
\text { Test: } 46.8\end{array}$ \\
\hline$[37]$ & Xboost & 195 & $\begin{array}{l}\text { BraTS } \\
2017\end{array}$ & Valid:50 \\
\hline
\end{tabular}

\section{Limitations of tumor segmentation and OS prediction}

The tumor segmentation result depends on the architectural design (from shallow CNN to the ensemble/cascaded
$\mathrm{CNN}$ ), amount of training data, input pre-processing, type of input(2D/3D), network optimization as well as post-processing of the generated output. Still, the DL methods have certain limitations, which include:

- Over-fitting: The common problem of the DNN based approach is over-fitting. It may occur due to the unavailability of the ample amount of labeled training data for brain tumor segmentation, which refers to a model that has an excellent performance on the training dataset but does not perform well on new data. The over-fitting problem can be handled either by reducing the network complexity (in terms of network layers and parameters) or by generating an ample amount of training data using image augmentation techniques. The augmentation techniques produce new images by performing data transformations and the corresponding ground truth that includes operations of scaling, rotation, translation, brightness variation, elastic deformations, horizontal flipping, and mirroring.

- Class imbalance: Class imbalance is another issue in tumor segmentation where the background class dominates the foreground class(tumor). Class imbalance can be handle by proper training data sampling, improved loss functions, and augmentation techniques.

The highest reported accuracy for the survival prediction task does not exceed $63 \%$ [45]. It is due to the dependency on the extraction of the features on the segmentation results. The incorrect segmentation results in wrong feature extraction for survival prediction. The biological importance of the extracted features also plays an important role. If the relevance of the features is 3 not known correctly, the survival prediction cannot be accurate. Besides, the importance of tumor sub-regions plays a role in feature extraction. The dataset includes all the pre-operative scans and does not give any other information like the success of tumor removal, postoperative treatments to the patients, and the response of the patients to such treatments. Chances of tumor reoccurrence are high if the patient is exposed to the radiation environment. Features related to it may further improve the OS prediction.

\section{Discussion and Future Direction}

The availability of benchmark dataset(BraTS) has grown the field of computer-assisted medical image analysis for brain tumor segmentation. The article covers detailed literature survey for brain tumor segmentation techniques. The tumor segmentation is approached with various techniques like semi-automated and automated 
methods. The semi-automated methods works on the input provided by user. Such methods suffer from limitations like manual seed point selection, atlas creation. Methods were gradually improved to include machine learning techniques like clustering, RF, ANN. The limitation of such methods is the selection of the features for training, which requires the knowledge of biological information of the image. The need of domain knowledge is removed by CNNs - deep neural network. CNN extracts high level features at deeper layers successively from the low level features from the preceding layers. This feature learning has improved the performance of CNN for tumor segmentation. The improvements to CNN are its variants, FCNN and ensemble of CNN/FCNN. The detailing of all the methods along with pre-processing, post-processing, prominent highlight of the methods and evaluation measure is given in Table 5, Table 8 , Table 10 and Table 11.

The ensemble generates robust segmentation results as well as improvements in the accuracy of the network. All these methods generate spurious segmentation results, which improves with the help of post-processing techniques like connected component analysis, spatial regularization and morphological operations to fine-tune the output. The class imbalance is the primary concern in the training of CNN for medical image analysis. The balanced input selection and loss function for a positive class will resolve the issue. Due to the unavailability of less amount of data for training, it may be possible that the network overfits the training data. The regularization and dropout resolve such issues. Moreover, as Bayesian CNN[127] handles epistemic and aleatoric uncertainties in the presence of limited data and knowledge, such type of network is useful for semantic segmentation. Adaptive loss[15], approximates varieties of loss functions with single latent variable. This function can further be thought of to solve the problem of semantic segmentation. Despite the popularity such methods have following limitations:

- computational efficiency

- memory requirement: As the depth of the network increases, the number of network parameters increases. This increase in the parameter requires additional memory as well as time to tune those on each epoch.

- Ample amount of annotated training data requirement: The annotated data generation is itself a challenge as this process is very time consuming and the annotation results may change depending on variability of the expert. In addition specific annotation tools are required by the expert for proper delineation and annotation purpose. In place of voxel annotation, image labeling for presence/absence of tumor is less time consuming, does not require much expertise and specialized annotation tool. Use of these image labeling in addition to the voxel labeling may help the network to learn relevant features for segmentation with less annotated data.

Acknowledgements The authors would like to thank NVIDIA Corporation for donating the Quadro K5200 and Quadro P5000 GPU used for this research, Dr. Krutarth Agravat (Medical Officer, Essar Ltd) for clearing our doubts related to medical concepts, Po-yu Kao, and Ujjawal Baid for their continuous support and help, Dr. Spyros and his entire team for BraTS dataset. The authors acknowledge continuous support from Professor Sanjay Chaudhary, Professor N. Padmanabhan, and Professor Manjunath Joshi for this work.

\section{References}

1. Abadi M, Barham P, Chen J, Chen Z, Davis A, Dean J, Devin M, Ghemawat S, Irving G, Isard M, et al. (2016) Tensorflow: A system for large-scale machine learning. In: 12th $\{$ USENIX\} Symposium on Operating Systems Design and Implementation (\{OSDI\} 16), pp 265-283

2. Agn M, Puonti O, af Rosenschöld PM, Law I, Van Leemput K (2015) Brain tumor segmentation using a generative model with an rbm prior on tumor shape. In: BrainLes 2015, Springer, pp 168-180

3. Agravat R, Raval MS (2019) Brain tumor segmentation and survival prediction. arXiv preprint arXiv:190909399

4. Agravat RR, Raval MS (2016) Brain tumor segmentation. CSI Communication

5. Agravat RR, Raval MS (2018) Deep learning for automated brain tumor segmentation in mri images. In: Soft Computing Based Medical Image Analysis, Elsevier, pp 183-201

6. Agravat RR, Raval MS (2019) Prediction of overall survival of brain tumor patients. In: TENCON 2019-2019 IEEE Region 10 Conference (TENCON), IEEE, pp 31-35

7. Albiol A, Albiol A, Albiol F (2018) Extending 2d deep learning architectures to $3 \mathrm{~d}$ image segmentation problems. In: International MICCAI Brainlesion Workshop, Springer, pp 73-82

8. Badrinarayanan V, Kendall A, Cipolla R (2017) Segnet: A deep convolutional encoder-decoder architecture for image segmentation. IEEE transactions on pattern analysis and machine intelligence 39(12):2481-2495

9. Baid U, Talbar S, Rane S, Gupta S, Thakur MH, Moiyadi A, Thakur S, Mahajan A (2018) Deep learning radiomics algorithm for gliomas (drag) model: a novel approach using $3 \mathrm{~d}$ unet based deep 
convolutional neural network for predicting survival in gliomas. In: International MICCAI Brainlesion Workshop, Springer, pp 369-379

10. Bakas S, Zeng K, Sotiras A, Rathore S, Akbari H, Gaonkar B, Rozycki M, Pati S, Davatzikos C (2015) Glistrboost: combining multimodal mri segmentation, registration, and biophysical tumor growth modeling with gradient boosting machines for glioma segmentation. In: BrainLes 2015, Springer, pp 144-155

11. Bakas S, Akbari H, Sotiras A, Bilello M, Rozycki M, Kirby J, Freymann J, Farahani K, Davatzikos C (2017) Segmentation labels and radiomic features for the pre-operative scans of the tcga-gbm collection. the cancer imaging archive (2017)

12. Bakas S, Akbari H, Sotiras A, Bilello M, Rozycki M, Kirby J, Freymann J, Farahani K, Davatzikos C (2017) Segmentation labels and radiomic features for the pre-operative scans of the tcga-lgg collection. The Cancer Imaging Archive 286

13. Bakas S, Akbari H, Sotiras A, Bilello M, Rozycki M, Kirby JS, Freymann JB, Farahani K, Davatzikos C (2017) Advancing the cancer genome atlas glioma mri collections with expert segmentation labels and radiomic features. Scientific data 4:170117

14. Banerjee S, Mitra S, Shankar BU (2018) Multiplanar spatial-convnet for segmentation and survival prediction in brain cancer. In: International MICCAI Brainlesion Workshop, Springer, pp 94104

15. Barron JT (2019) A general and adaptive robust loss function. In: Proceedings of the IEEE Conference on Computer Vision and Pattern Recognition, pp 4331-4339

16. Bastien F, Lamblin P, Pascanu R, Bergstra J, Goodfellow I, Bergeron A, Bouchard N, WardeFarley D, Bengio Y (2012) Theano: new features and speed improvements. arXiv preprint arXiv:12115590

17. Bauer S, Fejes T, Slotboom J, Wiest R, Nolte LP, Reyes M (2012) Segmentation of brain tumor images based on integrated hierarchical classification and regularization. In: MICCAI BraTS Workshop. Nice: Miccai Society, p 11

18. Bernal J, Kushibar K, Asfaw DS, Valverde S, Oliver A, Martí R, Lladó X (2019) Deep convolutional neural networks for brain image analysis on magnetic resonance imaging: a review. Artificial intelligence in medicine 95:64-81

19. Bharath HN, Colleman S, Sima DM, Van Huffel S (2017) Tumor segmentation from multimodal mri using random forest with superpixel and tensor based feature extraction. In: International MICCAI Brainlesion Workshop, Springer, pp 463-473

20. Buendia P, Taylor T, Ryan M, John N (2013) A grouping artificial immune network for segmentation of tumor images. Multimodal Brain Tumor Segmentation 1

21. Casamitjana A, Puch S, Aduriz A, Vilaplana V (2016) 3d convolutional neural networks for brain tumor segmentation: a comparison of multiresolution architectures. In: International Workshop on Brainlesion: Glioma, Multiple Sclerosis, Stroke and Traumatic Brain Injuries, Springer, pp 150-161

22. Casamitjana A, Catà M, Sánchez I, Combalia M, Vilaplana V (2017) Cascaded v-net using roi masks for brain tumor segmentation. In: International MICCAI Brainlesion Workshop, Springer, pp 381-391

23. Castillo LS, Daza LA, Rivera LC, Arbeláez P (2017) Brain tumor segmentation and parsing on mris using multiresolution neural networks. In: International MICCAI Brainlesion Workshop, Springer, pp 332-343

24. Center RM (2019 (accessed April 6, 2020)) Health Encyclopedia. URL https://www.urmc.rochester.edu/encyclopedia/content.aspx

25. Chandra S, Vakalopoulou M, Fidon L, Battistella E, Estienne T, Sun R, Robert C, Deutsch E, Paragios N (2019) Context aware 3d cnns for brain tumor segmentation. brainles 2018. Springer LNCS 11384:299-310

26. Chang PD (2016) Fully convolutional deep residual neural networks for brain tumor segmentation. In: International Workshop on Brainlesion: Glioma, Multiple Sclerosis, Stroke and Traumatic Brain Injuries, Springer, pp 108-118

27. Chen L, Bentley P, Mori K, Misawa K, Fujiwara M, Rueckert D (2018) Drinet for medical image segmentation. IEEE transactions on medical imaging 37(11):2453-2462

28. Chen LC, Papandreou G, Kokkinos I, Murphy K, Yuille AL (2017) Deeplab: Semantic image segmentation with deep convolutional nets, atrous convolution, and fully connected crfs. IEEE transactions on pattern analysis and machine intelligence 40(4):834-848

29. Chen W, Liu B, Peng S, Sun J, Qiao X (2018) S3d-unet: separable 3d u-net for brain tumor segmentation. In: International MICCAI Brainlesion Workshop, Springer, pp 358-368

30. Chollet F, et al. (2018) Keras: The python deep learning library. Astrophysics Source Code Library 
31. Choudhury AR, Vanguri R, Jambawalikar SR, Kumar P (2018) Segmentation of brain tumors using deeplabv3+. In: International MICCAI Brainlesion Workshop, Springer, pp 154-167

32. Clark MC, Hall LO, Goldgof DB, Velthuizen R, Murtagh FR, Silbiger MS (1998) Automatic tumor segmentation using knowledge-based techniques. IEEE transactions on medical imaging 17(2):187-201

33. Colmeiro RR, Verrastro C, Grosges T (2017) Multimodal brain tumor segmentation using $3 \mathrm{~d}$ convolutional networks. In: International MICCAI Brainlesion Workshop, Springer, pp 226-240

34. Cordier N, Menze B, Delingette H, Ayache N (2013) Patch-based segmentation of brain tissues

35. Corso JJ, Sharon E, Dube S, El-Saden S, Sinha U, Yuille A (2008) Efficient multilevel brain tumor segmentation with integrated bayesian model classification. IEEE transactions on medical imaging 27(5):629-640

36. Cuadra MB, Pollo C, Bardera A, Cuisenaire O, Villemure JG, Thiran JP (2004) Atlas-based segmentation of pathological $\mathrm{mr}$ brain images using a model of lesion growth. IEEE transactions on medical imaging 23(10):1301-1314

37. Dai L, Li T, Shu H, Zhong L, Shen H, Zhu H (2018) Automatic brain tumor segmentation with domain adaptation. In: International MICCAI Brainlesion Workshop, Springer, pp 380-392

38. Dera D, Raman F, Bouaynaya N, FathallahShaykh HM (2016) Interactive semi-automated method using non-negative matrix factorization and level set segmentation for the brats challenge. In: International Workshop on Brainlesion: Glioma, Multiple Sclerosis, Stroke and Traumatic Brain Injuries, Springer, pp 195-205

39. Dieleman S, Schlüter J, Raffel C, Olson E, Sønderby SK, Nouri D, et al. (2015) Lasagne: First release. DOI 10.5281/zenodo.27878, URL http://dx.doi.org/10.5281/zenodo.27878

40. Dong H, Supratak A, Mai L, Liu F, Oehmichen A, Yu S, Guo Y (2017) Tensorlayer: a versatile library for efficient deep learning development. In: Proceedings of the 25th ACM international conference on Multimedia, pp 1201-1204

41. Dong H, Yang G, Liu F, Mo Y, Guo Y (2017) Automatic brain tumor detection and segmentation using u-net based fully convolutional networks. In: annual conference on medical image understanding and analysis, Springer, pp 506-517

42. Doyle S, Vasseur F, Dojat M, Forbes F (2013) Fully automatic brain tumor segmentation from multiple mr sequences using hidden markov fields and variational em. Procs NCI-MICCAI BraTS pp 18-22

43. Ellwaa A, Hussein A, AlNaggar E, Zidan M, Zaki M, Ismail MA, Ghanem NM (2016) Brain tumor segmantation using random forest trained on iteratively selected patients. In: International Workshop on Brainlesion: Glioma, Multiple Sclerosis, Stroke and Traumatic Brain Injuries, Springer, pp 129-137

44. Feng X, Meyer C (2017) Patch-based 3d u-net for brain tumor segmentation. In: International Conference on Medical Image Computing and Computer-Assisted Intervention (MICCAI)

45. Feng X, Tustison N, Meyer C (2018) Brain tumor segmentation using an ensemble of $3 \mathrm{~d}$ u-nets and overall survival prediction using radiomic features. In: International MICCAI Brainlesion Workshop, Springer, pp 279-288

46. Festa J, Pereira S, Mariz JA, Sousa N, Silva CA (2013) Automatic brain tumor segmentation of multi-sequence $\mathrm{mr}$ images using random decision forests. Proceedings of NCI-MICCAI BRATS 1:23-26

47. Geremia E, Menze BH, Ayache N, et al. (2012) Spatial decision forests for glioma segmentation in multi-channel $\mathrm{mr}$ images. MICCAI Challenge on Multimodal Brain Tumor Segmentation 34

48. Goetz M, Weber C, Bloecher J, Stieltjes B, Meinzer HP, Maier-Hein K (2014) Extremely randomized trees based brain tumor segmentation. Proceeding of BRATS challenge-MICCAI pp 006011

49. Goyal B, Agrawal S, Sohi B (2018) Noise issues prevailing in various types of medical images. Biomedical \& Pharmacology Journal 11(3):1227

50. Guo X, Schwartz L, Zhao B (2013) Semiautomatic segmentation of multimodal brain tumor using active contours. Multimodal Brain Tumor Segmentation 27

51. Hamamci A, Unal G (2012) Multimodal brain tumor segmentation using the tumor-cut method on the brats dataset. Proc MICCAI-BRATS pp 1923

52. Hamamci A, Kucuk N, Karaman K, Engin K, Unal G (2011) Tumor-cut: segmentation of brain tumors on contrast enhanced $\mathrm{mr}$ images for radiosurgery applications. IEEE transactions on medical imaging 31(3):790-804

53. Havaei M, Dutil F, Pal C, Larochelle H, Jodoin PM (2015) A convolutional neural network approach to brain tumor segmentation. In: BrainLes 2015, Springer, pp 195-208 
54. Havaei M, Davy A, Warde-Farley D, Biard A, Courville A, Bengio Y, Pal C, Jodoin PM, Larochelle H (2017) Brain tumor segmentation with deep neural networks. Medical image analysis $35: 18-31$

55. Healthcareplex (2016 (accessed April 10, 2020)) CT Scan vs. MRI. URL https://healthcareplex.com/mri-vs-ct-scan/

56. Hopkins J (2019 (accessed April 6, 2020)) Health. URL https://www.hopkinsmedicine.org/health/conditionsand-diseases/basics-of-brain-tumors

57. Hu X, Li H, Zhao Y, Dong C, Menze BH, Piraud $\mathrm{M}$ (2018) Hierarchical multi-class segmentation of glioma images using networks with multilevel activation function. In: International MICCAI Brainlesion Workshop, Springer, pp 116-127

58. Hu Y, Xia Y (2017) 3d deep neural network-based brain tumor segmentation using multimodality magnetic resonance sequences. In: International MICCAI Brainlesion Workshop, Springer, pp 423434

59. Hu Y, Liu X, Wen X, Niu C, Xia Y (2018) Brain tumor segmentation on multimodal $\mathrm{mr}$ imaging using multi-level upsampling in decoder. In: International MICCAI Brainlesion Workshop, Springer, pp 168-177

60. Hua R, Huo Q, Gao Y, Sun Y, Shi F (2018) Multimodal brain tumor segmentation using cascaded v-nets. In: International MICCAI Brainlesion Workshop, Springer, pp 49-60

61. Huang G, Liu Z, Van Der Maaten L, Weinberger KQ (2017) Densely connected convolutional networks. In: Proceedings of the IEEE conference on computer vision and pattern recognition, pp 47004708

62. Isensee F, Kickingereder P, Wick W, Bendszus M, Maier-Hein KH (2017) Brain tumor segmentation and radiomics survival prediction: Contribution to the brats 2017 challenge. In: International MICCAI Brainlesion Workshop, Springer, pp 287-297

63. Isensee F, Kickingereder P, Wick W, Bendszus M, Maier-Hein KH (2018) No new-net. In: International MICCAI Brainlesion Workshop, Springer, pp 234-244

64. Islam M, Ren H (2017) Multi-modal pixelnet for brain tumor segmentation. In: International MICCAI Brainlesion Workshop, Springer, pp 298-308

65. Islam M, Jose VJM, Ren H (2018) Glioma prognosis: Segmentation of the tumor and survival prediction using shape, geometric and clinical information. In: International MICCAI Brainlesion Workshop, Springer, pp 142-153
66. Janssen PM, Hoff EI (2012) Teaching neuroimages: Subacute intracerebral hemorrhage mimicking brain tumor. Neurology 79(21):e183-e183

67. Jesson A, Arbel T (2017) Brain tumor segmentation using a $3 \mathrm{~d}$ fcn with multi-scale loss. In: International MICCAI Brainlesion Workshop, Springer, pp 392-402

68. Jia Y, Shelhamer E, Donahue J, Karayev S, Long J, Girshick R, Guadarrama S, Darrell T (2014) Caffe: Convolutional architecture for fast feature embedding. In: Proceedings of the 22nd ACM international conference on Multimedia, pp 675-678

69. Jungo A, McKinley R, Meier R, Knecht U, Vera L, Pérez-Beteta J, Molina-García D, PérezGarcía VM, Wiest R, Reyes M (2017) Towards uncertainty-assisted brain tumor segmentation and survival prediction. In: International MICCAI Brainlesion Workshop, Springer, pp 474-485

70. Kamnitsas K, Ferrante E, Parisot S, Ledig C, Nori AV, Criminisi A, Rueckert D, Glocker B (2016) Deepmedic for brain tumor segmentation. In: International workshop on Brainlesion: Glioma, multiple sclerosis, stroke and traumatic brain injuries, Springer, pp 138-149

71. Kamnitsas K, Bai W, Ferrante E, McDonagh S, Sinclair M, Pawlowski N, Rajchl M, Lee M, Kainz B, Rueckert D, et al. (2017) Ensembles of multiple models and architectures for robust brain tumour segmentation. In: International MICCAI Brainlesion Workshop, Springer, pp 450-462

72. Kao PY, Ngo T, Zhang A, Chen JW, Manjunath B (2018) Brain tumor segmentation and tractographic feature extraction from structural $\mathrm{mr}$ images for overall survival prediction. In: International MICCAI Brainlesion Workshop, Springer, pp 128-141

73. Kermi A, Mahmoudi I, Khadir MT (2018) Deep convolutional neural networks using u-net for automatic brain tumor segmentation in multimodal mri volumes. In: International MICCAI Brainlesion Workshop, Springer, pp 37-48

74. Kim G (2017) Brain tumor segmentation using deep fully convolutional neural networks. In: International MICCAI Brainlesion Workshop, Springer, pp 344-357

75. Kleesiek J, Biller A, Urban G, Kothe U, Bendszus M, Hamprecht F (2014) Ilastik for multi-modal brain tumor segmentation. Proceedings MICCAI BraTS (brain tumor segmentation challenge) pp 12-17

76. Kori A, Soni M, Pranjal B, Khened M, Alex V, Krishnamurthi G (2018) Ensemble of fully convolutional neural network for brain tumor segmenta- 
tion from magnetic resonance images. In: International MICCAI Brainlesion Workshop, Springer, pp $485-496$

77. Krizhevsky A, Sutskever I, Hinton GE (2012) Imagenet classification with deep convolutional neural networks. In: Advances in neural information processing systems, pp 1097-1105

78. Kwon D, Akbari H, Da X, Gaonkar B, Davatzikos C (2014) Multimodal brain tumor image segmentation using glistr. MICCAI brain tumor segmentation (BraTS) challenge manuscripts pp 18-19

79. Lachinov D, Vasiliev E, Turlapov V (2018) Glioma segmentation with cascaded unet. In: International MICCAI Brainlesion Workshop, Springer, pp 189-198

80. Le Folgoc L, Nori AV, Ancha S, Criminisi A (2016) Lifted auto-context forests for brain tumour segmentation. In: International Workshop on Brainlesion: Glioma, Multiple Sclerosis, Stroke and Traumatic Brain Injuries, Springer, pp 171-183

81. LeCun Y, Bottou L, Bengio Y, Haffner P (1998) Gradient-based learning applied to document recognition. Proceedings of the IEEE 86(11):22782324

82. Lefkovits L, Lefkovits S, Szilágyi L (2016) Brain tumor segmentation with optimized random forest. In: International Workshop on Brainlesion: Glioma, Multiple Sclerosis, Stroke and Traumatic Brain Injuries, Springer, pp 88-99

83. Li Y, Shen L (2017) Deep learning based multimodal brain tumor diagnosis. In: International MICCAI Brainlesion Workshop, Springer, pp 149158

84. Lin TY, Goyal P, Girshick R, He K, Dollár P (2017) Focal loss for dense object detection. In: Proceedings of the IEEE international conference on computer vision, pp 2980-2988

85. Long J, Shelhamer E, Darrell T (2015) Fully convolutional networks for semantic segmentation. In: Proceedings of the IEEE conference on computer vision and pattern recognition, pp 3431-3440

86. Lopez MM, Ventura J (2017) Dilated convolutions for brain tumor segmentation in mri scans. In: International MICCAI Brainlesion Workshop, Springer, pp 253-262

87. Ma J, Yang X (2018) Automatic brain tumor segmentation by exploring the multi-modality complementary information and cascaded $3 \mathrm{~d}$ lightweight cnns. In: International MICCAI Brainlesion Workshop, Springer, pp 25-36

88. Maier O, Wilms M, Handels H (2015) Image features for brain lesion segmentation using random forests. In: BrainLes 2015, Springer, pp 119-130
89. Marcinkiewicz M, Nalepa J, Lorenzo PR, Dudzik W, Mrukwa G (2018) Segmenting brain tumors from mri using cascaded multi-modal u-nets. In: International MICCAI Brainlesion Workshop, Springer, pp 13-24

90. McKinley R, Wepfer R, Gundersen T, Wagner F, Chan A, Wiest R, Reyes M (2016) Nablanet: A deep dag-like convolutional architecture for biomedical image segmentation. In: International Workshop on Brainlesion: Glioma, Multiple Sclerosis, Stroke and Traumatic Brain Injuries, Springer, pp 119-128

91. McKinley R, Jungo A, Wiest R, Reyes M (2017) Pooling-free fully convolutional networks with dense skip connections for semantic segmentation, with application to brain tumor segmentation. In: International MICCAI Brainlesion Workshop, Springer, pp 169-177

92. McKinley R, Meier R, Wiest R (2018) Ensembles of densely-connected cnns with label-uncertainty for brain tumor segmentation. In: International MICCAI Brainlesion Workshop, Springer, pp 456465

93. Media H (2004 (accessed April 10, 2020)) CT Scan vs. MRI. URL https://www.healthline.com/health/ct-scanvs-mri/

94. Mehta R, Arbel T (2018) 3d u-net for brain tumour segmentation. In: International MICCAI Brainlesion Workshop, Springer, pp 254-266

95. Meier R, Bauer S, Slotboom J, Wiest R, Reyes M (2013) A hybrid model for multimodal brain tumor segmentation. Multimodal Brain Tumor Segmentation 31:31-37

96. Meier R, Bauer S, Slotboom J, Wiest R, Reyes M (2014) Appearance-and context-sensitive features for brain tumor segmentation. Proceedings of MICCAI BRATS Challenge pp 020-026

97. Meier R, Karamitsou V, Habegger S, Wiest R, Reyes M (2015) Parameter learning for crf-based tissue segmentation of brain tumors. In: BrainLes 2015, Springer, pp 156-167

98. Meier R, Knecht U, Wiest R, Reyes M (2016) Crf-based brain tumor segmentation: alleviating the shrinking bias. In: International workshop on brainlesion: glioma, multiple sclerosis, stroke and traumatic brain injuries, Springer, pp 100-107

99. Menze BH, Geremia E, Ayache N, Szekely G (2012) Segmenting glioma in multi-modal images using a generative-discriminative model for brain lesion segmentation. Proc MICCAI-BRATS (Multimodal Brain Tumor Segmentation Challenge) 8 
100. Menze BH, Jakab A, Bauer S, Kalpathy-Cramer J, Farahani K, Kirby J, Burren Y, Porz N, Slotboom J, Wiest R, et al. (2014) The multimodal brain tumor image segmentation benchmark (brats). IEEE transactions on medical imaging 34(10):1993-2024

101. Myronenko A (2018) 3d mri brain tumor segmentation using autoencoder regularization. In: International MICCAI Brainlesion Workshop, Springer, pp 311-320

102. Nuechterlein N, Mehta S (2019) 3d-espnet with pyramidal refinement for volumetric brain tumor image segmentation. brainles 2018. Springer LNCS 11384:245-253

103. Nvidia (2020 (accessed April 28, 2020)) Nvidia. URL https://www.nvidia.com/en-in/

104. Nyúl LG, Udupa JK (1999) On standardizing the $\mathrm{mr}$ image intensity scale. Magnetic Resonance in Medicine: An Official Journal of the International Society for Magnetic Resonance in Medicine 42(6):1072-1081

105. Pan SJ, Yang Q (2009) A survey on transfer learning. IEEE Transactions on knowledge and data engineering 22(10):1345-1359

106. Paszke A, Gross S, Massa F, Lerer A, Bradbury J, Chanan G, Killeen T, Lin Z, Gimelshein N, Antiga L, et al. (2019) Pytorch: An imperative style, highperformance deep learning library. In: Advances in Neural Information Processing Systems, pp 80248035

107. Pawar K, Chen Z, Shah NJ, Egan G (2017) Residual encoder and convolutional decoder neural network for glioma segmentation. In: International MICCAI Brainlesion Workshop, Springer, pp 263273

108. Pereira S, Pinto A, Alves V, Silva CA (2015) Deep convolutional neural networks for the segmentation of gliomas in multi-sequence mri. In: BrainLes 2015, Springer, pp 131-143

109. Phophalia A, Maji P (2017) Multimodal brain tumor segmentation using ensemble of forest method. In: International MICCAI Brainlesion Workshop, Springer, pp 159-168

110. Piedra EAR, Ellingson BM, Taira RK, El-Saden S, Bui AA, Hsu W (2016) Brain tumor segmentation by variability characterization of tumor boundaries. In: International Workshop on Brainlesion: Glioma, Multiple Sclerosis, Stroke and Traumatic Brain Injuries, Springer, pp 206-216

111. Pourreza R, Zhuge Y, Ning H, Miller R (2017) Brain tumor segmentation in mri scans using deeply-supervised neural networks. In: International MICCAI Brainlesion Workshop, Springer, pp 320-331

112. Prastawa M, Bullitt E, Ho S, Gerig G (2004) A brain tumor segmentation framework based on outlier detection. Medical image analysis 8(3):275-283

113. Puch S, Sánchez I, Hernández A, Piella G, Prćkovska V (2019) Global planar convolutions for improved context aggregation in brain tumor segmentation. brainles 2018. Springer LNCS 11384:393-405

114. Puybareau E, Tochon G, Chazalon J, Fabrizio J (2018) Segmentation of gliomas and prediction of patient overall survival: a simple and fast procedure. In: International MICCAI Brainlesion Workshop, Springer, pp 199-209

115. of Radiology AC (1999 (accessed April 10, 2020)) Brain Tumor Treatment. URL https://www.radiologyinfo.org/

116. Rajendran A, Dhanasekaran R (2012) Brain tumor segmentation on mri brain images with fuzzy clustering and gvf snake model. International Journal of Computers Communications \& Control 7(3):530-539

117. Randhawa RS, Modi A, Jain P, Warier P (2016) Improving boundary classification for brain tumor segmentation and longitudinal disease progression. In: International Workshop on Brainlesion: Glioma, Multiple Sclerosis, Stroke and Traumatic Brain Injuries, Springer, pp 65-74

118. Raviv TR, Leemput KV, Menze BH (2012) Multimodal brain tumor segmentation via latent atlases. Proceeding MICCAIBRATS 64

119. Reza S, Iftekharuddin K (2013) Multi-class abnormal brain tissue segmentation using texture. Multimodal Brain Tumor Segmentation 38

120. Reza S, Iftekharuddin K (2014) Improved brain tumor tissue segmentation using texture features. Proceedings MICCAI BraTS (brain tumor segmentation challenge) pp 27-30

121. Ronneberger O, Fischer P, Brox T (2015) U-net: Convolutional networks for biomedical image segmentation. In: International Conference on Medical image computing and computer-assisted intervention, Springer, pp 234-241

122. Saha R, Phophalia A, Mitra SK (2016) Brain tumor segmentation from multimodal $\mathrm{mr}$ images using rough sets. In: International Conference on Computer Vision, Graphics, and Image processing, Springer, pp 133-144

123. Serrano-Rubio J, Everson R (2019) Brain tumour segmentation method based on supervoxels and sparse dictionaries. brainles 2018. Springer LNCS 11384:210-221 
124. Shaikh M, Anand G, Acharya G, Amrutkar A, Alex V, Krishnamurthi G (2017) Brain tumor segmentation using dense fully convolutional neural network. In: International MICCAI Brainlesion Workshop, Springer, pp 309-319

125. Shboul ZA, Vidyaratne L, Alam M, Iftekharuddin KM (2017) Glioblastoma and survival prediction. In: International MICCAI Brainlesion Workshop, Springer, pp 358-368

126. Shin HC (2012) Hybrid clustering and logistic regression for multi-modal brain tumor segmentation. In: Proc. of Workshops and Challanges in Medical Image Computing and ComputerAssisted Intervention (MICCAI'12)

127. Shridhar K, Laumann F, Liwicki M (2019) A comprehensive guide to bayesian convolutional neural network with variational inference. arXiv preprint arXiv:190102731

128. Soltaninejad M, Zhang L, Lambrou T, Yang G, Allinson N, Ye X (2017) Mri brain tumor segmentation and patient survival prediction using random forests and fully convolutional networks. In: International MICCAI Brainlesion Workshop, Springer, pp 204-215

129. Song B, Chou CR, Chen X, Huang A, Liu MC (2016) Anatomy-guided brain tumor segmentation and classification. In: International Workshop on Brainlesion: Glioma, Multiple Sclerosis, Stroke and Traumatic Brain Injuries, Springer, pp 162170

130. Stawiaski J (2018) A pretrained densenet encoder for brain tumor segmentation. In: International MICCAI Brainlesion Workshop, Springer, pp 105115

131. Subbanna N, Arbel T (2012) Probabilistic gabor and markov random fields segmentation of brain tumours in mri volumes. Proc MICCAI Brain Tumor Segmentation Challenge (BRATS) pp 28-31

132. Sudre CH, Li W, Vercauteren T, Ourselin S, Cardoso MJ (2017) Generalised dice overlap as a deep learning loss function for highly unbalanced segmentations. In: Deep learning in medical image analysis and multimodal learning for clinical decision support, Springer, pp 240-248

133. Sun L, Zhang S, Luo L (2018) Tumor segmentation and survival prediction in glioma with deep learning. In: International MICCAI Brainlesion Workshop, Springer, pp 83-93

134. Szegedy C, Liu W, Jia Y, Sermanet P, Reed S, Anguelov D, Erhan D, Vanhoucke V, Rabinovich A (2015) Going deeper with convolutions. In: Proceedings of the IEEE conference on computer vision and pattern recognition, pp 1-9
135. Szegedy C, Vanhoucke V, Ioffe S, Shlens J, Wojna Z (2016) Rethinking the inception architecture for computer vision. In: Proceedings of the IEEE conference on computer vision and pattern recognition, pp 2818-2826

136. Targ S, Almeida D, Lyman K (2016) Resnet in resnet: Generalizing residual architectures. arXiv preprint arXiv:160308029

137. Taylor T, John N, Buendia P, Ryan M (2013) Map-reduce enabled hidden markov models for high throughput multimodal brain tumor segmentation. Multimodal Brain Tumor Segmentation 43

138. Tomas-Fernandez X, Wareld S (2012) Automatic brain tumor segmentation based on a coupled global-local intensity bayesian model. MICCAI Challenge on Multimodal Brain Tumor Segmentation 34

139. Tustison NJ, Avants BB, Cook PA, Zheng Y, Egan A, Yushkevich PA, Gee JC (2010) N4itk: improved n3 bias correction. IEEE transactions on medical imaging 29(6):1310-1320

140. Urban G, Bendszus M, Hamprecht F, Kleesiek J (2014) Multi-modal brain tumor segmentation using deep convolutional neural networks. MICCAI BraTS (brain tumor segmentation) challenge Proceedings, winning contribution pp 31-35

141. Van Griethuysen JJ, Fedorov A, Parmar C, Hosny A, Aucoin N, Narayan V, Beets-Tan RG, FillionRobin JC, Pieper S, Aerts HJ (2017) Computational radiomics system to decode the radiographic phenotype. Cancer research 77(21):e104e107

142. Wang G, Li W, Ourselin S, Vercauteren T (2017) Automatic brain tumor segmentation using cascaded anisotropic convolutional neural networks. In: International MICCAI brainlesion workshop, Springer, pp 178-190

143. Wang G, Li W, Ourselin S, Vercauteren T (2018) Automatic brain tumor segmentation using convolutional neural networks with test-time augmentation. In: International MICCAI Brainlesion Workshop, Springer, pp 61-72

144. Weninger L, Rippel O, Koppers S, Merhof D (2018) Segmentation of brain tumors and patient survival prediction: Methods for the brats 2018 challenge. In: International MICCAI Brainlesion Workshop, Springer, pp 3-12

145. Xiao Y, Hu J (2012) Hierarchical random walker for multimodal brain tumor segmentation. MICCAI Challenge on Multimodal Brain Tumor Segmentation

146. Xu Y, Gong M, Fu H, Tao D, Zhang K, Batmanghelich K (2018) Multi-scale masked 3-d u-net 
for brain tumor segmentation. In: International MICCAI Brainlesion Workshop, Springer, pp 222233

147. Yao H, Zhou X, Zhang X (2019) Automatic segmentation of brain tumor using $3 \mathrm{~d}$ se-inception networks with residual connections. brainles 2018 . Springer LNCS 11384:346-357

148. Zeng K, Bakas S, Sotiras A, Akbari H, Rozycki M, Rathore S, Pati S, Davatzikos C (2016) Segmentation of gliomas in pre-operative and post-operative multimodal magnetic resonance imaging volumes based on a hybrid generativediscriminative framework. In: International Workshop on Brainlesion: Glioma, Multiple Sclerosis, Stroke and Traumatic Brain Injuries, Springer, pp 184-194

149. Zhao L, Wu W, Corso JJ (2012) Brain tumor segmentation based on gmm and active contour method with a model-aware edge map. BRATS MICCAI pp 19-23

150. Zhao L, Sarikaya D, Corso JJ (2013) Automatic brain tumor segmentation with mrf on supervoxels. Multimodal Brain Tumor Segmentation 51

151. Zhao X, Wu Y, Song G, Li Z, Fan Y, Zhang Y (2016) Brain tumor segmentation using a fully convolutional neural network with conditional random fields. In: International Workshop on Brainlesion: Glioma, Multiple Sclerosis, Stroke and Traumatic Brain Injuries, Springer, pp 75-87

152. Zhao X, Wu Y, Song G, Li Z, Zhang Y, Fan Y (2017) 3d brain tumor segmentation through integrating multiple $2 \mathrm{~d}$ fcnns. In: International MICCAI Brainlesion Workshop, Springer, pp 191-203

153. Zhou C, Chen S, Ding C, Tao D (2018) Learning contextual and attentive information for brain tumor segmentation. In: International MICCAI Brainlesion Workshop, Springer, pp 497-507

154. Zhou F, Li T, Li H, Zhu H (2017) Tpcnn: twophase patch-based convolutional neural network for automatic brain tumor segmentation and survival prediction. In: International MICCAI Brainlesion Workshop, Springer, pp 274-286

155. Zikic D, Glocker B, Konukoglu E, Criminisi A, Demiralp C, Shotton J, Thomas OM, Das T, Jena R, Price SJ (2012) Decision forests for tissuespecific segmentation of high-grade gliomas in multi-channel mr. In: International Conference on Medical Image Computing and ComputerAssisted Intervention, Springer, pp 369-376

156. Zikic D, Glocker B, Konukoglu E, Shotton J, Criminisi A, Ye D, Demiralp C, Thomas O, Das T, Jena R, et al. (2012) Context-sensitive classification forests for segmentation of brain tumor tis- sues. In: Proc. MICCAI-BRATS, pp 22-30

157. Zikic D, Ioannou Y, Brown M, Criminisi A (2014) Segmentation of brain tumor tissues with convolutional neural networks. Proceedings MICCAIBRATS pp 36-39 